\title{
Surface effects in nanoscale structures investigated by a fully-nonlocal energy-based quasicontinuum method
}

\author{
Jeffrey S. Amelang, Dennis M. Kochmann* \\ Graduate Aerospace Laboratories, California Institute of Technology, Pasadena, CA 91125, U.S.A.
}

\begin{abstract}
Surface effects in nanoscale mechanical systems such as nanoporous solids or small-scale structures can have a significant impact on the effective material response which deviates from the material behavior of bulk solids. Understanding such phenomena requires modeling techniques that locally retain atomistic information while transitioning to the relevant macroscopic length scales. We recently introduced a fully-nonlocal energy based quasicontinuum (QC) method equipped with new summation rules. This technique accurately bridges across scales from atomistics to the continuum through a thermodynamically-consistent coarse-graining scheme. Beyond minimizing energy approximation errors and spurious force artifacts, the new method also qualifies to describe free surfaces, which is reported here. Surfaces present a major challenge to coarse-grained atomistics, which has oftentimes been circumvented by costly ad-hoc extensions of the traditional QC method. We show that our new coarse-graining scheme successfully and automatically reduces spurious force artifacts near free surfaces. After discussing the computational model, we demonstrate its benefits in the presence of free surfaces by several nanomechanical examples including surface energy calculations, elastic size effects in nano-rods and in plates with nano-sized holes. Overall, we demonstrate the importance of surface effects as well as a new strategy to accurately capture those computationally via coarse-grained atomistics.
\end{abstract}

Keywords: surface, size effects, atomistics, quasicontinuum, multiscale modeling

\section{Introduction}

The mechanical behavior of nanoscale structures and nanostructured solids can deviate significantly from the material response observed in bulk solids. Example systems include micro- and nano-electro-mechanical devices and functional cellular solids with micro- and nano-sized morphological features, which promise tremendous technological and scientific potential. Nanoporous metals excel through unique physical, chemical, and mechanical characteristics (Biener et al., 2007), owing to the abundance of free surfaces. Depending on their degree of structural order, one distinguishes between nanoporous foams and structural materials (or, lattice materials). Both display high strength and stiffness and an extremely low mass density (as low as a few $\mathrm{mg} / \mathrm{cm}^{3}$ ). In addition, lattice materials offer unprecedented opportunities for bottom-up-engineered materials (Schaedler et al., 2011; Montemayor et al., 2014).

The extremely low spatial resolution of such systems is responsible for a variety of mechanical size effects. In particular, the abundance of free surfaces at the nanoscale alters the effective material behavior both within the reversible elastic regime and beyond. Surface relaxation alters the local atomic arrangement close to the surface, which in turn affects the atomic interactions and thus alters the effective elastic moduli. This size effect has been observed experimentally in single-crystalline nano-structures such as during elastic bending of nano-sized beams (Wong et al., 1997) or carbon nanotubes (Poncharal et al., 1999), as well as during tension-compression testing of nanowires, see e.g. (Chen et al., 2006; Agrawal et al., 2008; Asthana et al., 2011; Zhu et al., 2012; Chen et al., 2013). Since surface relaxation is confined to a few atomic layers, the elastic size effects become noticeable only at small wire diameters on the order of a few nanometers, whereas the bulk response dominates at larger sizes.

${ }^{*}$ Corresponding author (phone +1-626-395-8113, fax +1-626-395-2900).

Email address: kochmann@caltech.edu (Dennis M. Kochmann) 
Beyond the elastic limit, surfaces interact with lattice defects, e.g. by acting as dislocation and vacancy sinks and sources. Remarkable increases of the tensile strength of single-crystal metallic whiskers with decreasing whisker diameter were first reported in the pioneering work of Taylor (1924) and later confirmed by Brenner's studies (1956; 1957). Their research demonstrated what is now well-known as a structure-induced size effect in metals. Since then, there has been compelling experimental evidence that the strength of nano-sized single crystals exhibits a powerlaw dependence on the feature size (Zhu et al., 2009). Recent tension-compression experiments on metallic microand nano-pillars impressively demonstrated this extrinsic size effect, cf. (Greer et al., 2009; Burek and Greer, 2010; Kim et al., 2011). In particular, a variety of micro-deformation studies have revealed strong size effects in Cu single crystals (Dehm, 2009; Kiener et al., 2006, 2008, 2009; Maass et al., 2008; Jennings et al., 2010). At such small scales, three mechanisms play an important role: small volumes reduce the number of defects in a statistical fashion, and surfaces interact with defects. The increase in strength has been explained by dislocation starvation (Greer et al., 2005; Shan et al., 2008; Greer and Nix, 2006), source exhaustion (Rao et al., 2008; Norfleet et al., 2008), source truncation (Parthasarathy et al., 2007; Rao et al., 2007), or weakest-link mechanisms (Norfleet et al., 2008); see (Greer and De Hosson, 2011) for a review. In addition, surfaces alter the local stress state with important consequences for nano-scale structures, see e.g. (Gill, 2007; Pugno and Aifantis, 2011; Miri et al., 2011; Grekov and Yazovskaya, 2013).

From the modeling perspective, the study of nano-sized structural members is challenging. On the one hand, they reside outside the realm of traditional molecular dynamics (MD); computational costs severely limit the domain size that can be modeled as well as the range of viable strain rates (Derlet et al., 2003). On the other hand, size effects in nanoscale structures as well as surface-defect interactions hardly admit the use of the continuum hypothesis which forms the basis of most engineering models. Nonlocal and size-aware continuum models have been proposed to capture individual size effects at the nanoscale, see e.g. (Miller and Shenoy, 2000; Chen et al., 2006), yet they are commonly tailored for particular mechanisms and not universally applicable. Furthermore, the specific length scales involved in nanoscale structures oftentimes prohibit a separation of atomistic and structural scales. This calls for a powerful multiscale simulation methodology that bridges across scales and is capable of modeling nano- to micrometer-sized objects at the accuracy of the underlying atomistic ensemble.

Various methods have been developed to bridge the scales. On the one hand, hierarchical models are the method of choice when a clear separation of scales can be assumed so that homogenization techniques can extract the effective constitutive response from the atomistic scale and pass it to the structural level, see e.g. (Shephard et al., 2004; Chung, 2004; Park et al., 2005; Clayton and Chung, 2006; Liu et al., 2006). On the other hand, concurrent-scale coupling methods integrate different constitutive descriptions into a single-scale model by spatially separating domains treated e.g. by first-principles, MD, discrete defect mechanics, and continuum theories. Prominent examples comprise coupled atomistic/discrete-dislocation models such as CADD (Curtin and Miller, 2003; Shilkrot et al., 2004; Nair et al., 2010) and AtoDis (Brinckmann et al., 2012), furthermore the bridging domain method (Belytschko and Xiao, 2003) as well as MADD (Abraham et al., 1998; Broughton et al., 1999) which couples several scales. In such methods, a key challenge is the passing of information across interfaces between different model domains. Coarse-graining techniques circumvent this difficulty by applying the same lower-scale constitutive description to the entire model by up-scaling in space and/or in time. Examples include Coarse-Grained MD (Rudd and Broughton, 2005a,b) and the quasicontinuum (QC) method (Tadmor et al., 1996). While spatial and temporal coarse-graining are equally important, we here focus on coarse-graining in space. Thereby, the number of degrees of freedom is reduced by introducing geometric constraints, thus making the lower-scale accuracy available for efficient large-scale simulations, see (Tadmor et al., 1996; Suryanarayana, 2011; Iyer and Gavini, 2011) for examples. Coarse-graining in time (to transition from femtoseconds to minutes) can be added to the techniques investigated here but is beyond the scope of this paper; see e.g. (Voter, 1997, 1998; Sorensen and Voter, 2000; Voter et al., 2002; Kim et al., 2014; Venturini et al., 2014). Also, we focus on the response of crystalline ensembles at zero temperature. Finite temperature extensions can be applied to the presented spatial coarse-graining techniques, see e.g. (Shenoy et al., 1999b; Dupuy et al., 2005; Kulkarni et al., 2008; Marian et al., 2010; Ariza et al., 2012; Tadmor et al., 2013; Venturini et al., 2014).

Here, we concentrate on one such coarse-graining methodology, viz. the QC method, which enables us to extend the accuracy of an atomistic constitutive description to large sample sizes via an efficient coarse-graining technique. The remainder of this paper is organized as follows. Section 2 reviews the concepts and governing equations of the QC method, explains the new extensions of the model, and the particular challenges arising from free surfaces. Section 3 summarizes a series of numerical examples that demonstrate the benefits of the new method and the observed size effects, and Section 4 concludes our investigation. 


\section{The quasicontinuum method, summation rules, and free surfaces}

\subsection{The quasicontinuum method and its summation rules}

The QC method (Tadmor et al., 1996) is a popular numerical scheme to bridge the scales in crystalline solids from individual atoms to the macroscopic continuum. The energy-based QC technique (Eidel and Stukowski, 2009; Espanol et al., 2013; Amelang et al., 2015) achieves such coarse-graining based on three pillars which comprise (i) the interpolation of atomic positions from a carefully-chosen set of representative atoms (or repatoms for short), (ii) the approximation of thermodynamic quantities by means of summation rules which involve only a reduced set of lattice sites instead of the full atomistic ensemble, and (iii) model adaptation techniques which allow us to confine full atomistic resolution to lattice defects while efficiently coarse-graining in homogeneously-deforming regions away from those defects. Most QC flavors make use of an affine interpolation on a Delaunay-triangulated mesh, which will be used in the following. A large variety of summation rules have been introduced in the scientific literature, many of which were proposed in an ad-hoc manner to overcome QC specific deficiencies or limitations.

Similarly to rules of numerical quadrature used in the finite element method, summation rules, in a general sense, introduce a set of sampling atoms and associated sampling atom weights (in close analogy to quadrature points and associated weights). Based on those sampling atoms, in energy-based QC the total Hamiltonian (or the total potential energy for quasistatic problems) is approximated by a weighted sum over the respective quantities evaluated at the sampling atom sites. Popular summation rules include nodal and cluster rules, see e.g. (Eidel and Stukowski, 2009), quadrature-type and element-based summation rules, e.g. (Gunzburger and Zhang, 2010; Yang et al., 2013), and combinations thereof, see e.g. (Beex et al., 2014; Amelang et al., 2015). In contrast, force-based QC applies summation rules directly on the force level by approximating forces on repatoms by a weighted sum over sampling atoms (Knap and Ortiz, 2001). As discussed in (Amelang et al., 2015), this avoids residual force artifacts but comes with serious drawbacks such as its non-conservative framework and the associated lack of the existence of a potential energy with implications particularly for dynamics and finite temperature. Therefore, here and in the following we focus exclusively on the energy-based QC formulation.

In addition to the choice of repatoms used to interpolate atomic positions, the choice of a particular summation rule essentially affects the accuracy and efficiency of QC simulations. On the one hand, choosing large numbers of sampling atoms results in high accuracy. Yet, the large number of lattice sites involved in the calculation of energies and repatom forces leads to tremendous computational expenses which may defeat the purpose of coarsegrained atomistics. On the other hand, choosing very few sampling atoms considerably speeds up calculations and allows for the simulation of micron-sized problems (and above). However, this can result in large approximation errors, especially if the sampling atom locations and/or weights are chosen poorly. Furthermore, such approximation errors on the energy level propagate to the resulting interatomic forces and produce spurious force artifacts which are known as ghost forces in the local/non-local QC method, cf. (Miller et al., 1998; Shenoy et al., 1998; Dobson and Luskin, 2009), or as residual/spurious forces in the non-local energy-based QC method, see e.g. (Eidel and Stukowski, 2009; Iyer and Gavini, 2011). Residual forces on repatoms appear in the undeformed configuration when a summation rule is applied to non-uniform QC meshes. For example, in a full atomistic description every atom in an infinite (centrosymmetric) atomic ensemble undergoing affine deformations experiences no net force due to the symmetric cancellation of interatomic forces. However, as was shown in (Amelang et al., 2015), the most common QC summation rules (including nodal, cluster, and quadrature summation rules) produce noticeable residual force artifacts in affinely-strained non-uniform QC meshes. These apparent forces are non-physical and do not exist in the full atomistic scenario. Similarly, spurious forces arise in generally-deformed non-uniform QC meshes: the approximation made by a summation rule affects the computation of repatom forces, which then leads to significant errors in the simulation. Amelang et al. (2015) showed that ad-hoc corrections only solve a small part of the underlying problem. For example, the addition of dead loads to each repatom to correct for the residual forces in the undeformed configuration was shown to even exceed those errors obtained without the dead-load correction at sufficient levels of deformation. More involved ghost force corrections come with high numerical costs, see e.g. (Shenoy et al., 1999a).

Most previous studies of summation rules and the energy-based QC method have investigated approximation errors and force artifacts mainly by studying representative one- or two-dimensional examples using simplified potentials, oftentimes far from physical reality. In order to provide guidelines for the selection of optimal summation rules, we have conducted a comprehensive analysis of QC summation rules in three-dimensional examples involving millions of degrees of freedom and state-of-the-art interatomic potentials (Amelang et al., 2015). Based on a general formulation 
of summation rules, we compared traditional nodal, cluster, and quadrature summation rules with respect to their accuracy and efficiency, and we proposed new summation rules of first and second order which exhibit vanishing force artifacts and significantly smaller energy approximation errors in non-uniform QC meshes than all previous methods. To date, the benefits and shortcomings of the new scheme have not been assessed for the important problem of surfaces at the nanoscale and their impact on the mechanics of materials.

To evaluate the accuracy of the QC method, we compute solutions of complex boundary value problems using various QC summation rules and compare these to the exact solutions obtained from full lattice statics. The resulting total errors include (i) the aforementioned energy approximation errors due to the summation rules, (ii) errors arising from equilibration in the presence of force artifacts, and (iii) errors stemming from the QC mesh representation and its affine interpolation. In the elastic regime, a one-to-one comparison with full atomistics allows us to quantify those errors, whereas the formation of microstructure leads to statistical variations which complicates a direct comparison to atomistics (see e.g. (Amelang et al., 2015) for a comparison of inelastic deformation mechanisms via a statistical interpretation of results). In the following, we restrict our examples to zero-temperature quasistatic calculations, even though the general concepts apply equally to finite-temperature calculations as discussed above.

\subsection{Review of the basic governing equations}

What follows is a brief review of the non-local energy-based QC method in order to introduce our notation and the specific formulation of summation rules used in the following. The interested reader is referred to (Amelang et al., 2015 ) and the references therein for a more detailed description. The QC method introduces a set of $N_{h}$ representative atoms (or repatoms) with positions $\boldsymbol{x}(t)=\left\{\boldsymbol{x}_{1}(t), \ldots, \boldsymbol{x}_{N_{h}}(t)\right\}$ so that the positions $\boldsymbol{q}(t)=\left\{\boldsymbol{q}_{1}(t), \ldots, \boldsymbol{q}_{N}(t)\right\}$ of all $N$ lattice sites in a crystalline ensemble at time $t$ are obtained by interpolation via

$$
\boldsymbol{q}_{i} \approx \boldsymbol{q}_{i}^{h}=\sum_{a=1}^{N_{h}} N_{a}\left(\boldsymbol{X}_{i}\right) \boldsymbol{x}_{a}
$$

where $N_{a}\left(\boldsymbol{X}_{i}\right)$ is the shape function of repatom $a$ evaluated at the reference position $\boldsymbol{X}_{i}$ of lattice site $i$ in the undeformed lattice. We restrict our analysis to the most common interpolation scheme, viz. affine interpolation based on a Delaunay triangulation of the set of repatoms. In three dimensions, this leads to simplicial tetrahedral elements with linear shape functions.

In crystalline solids such as metals and ceramics we commonly find an additive decomposition of the total potential energy $V$ into contributions from all lattice sites. In order to further reduce computational complexity, energy-based summation rules thus approximate the total Hamiltonian of the crystal lattice by a weighted sum over the set of $N_{s}$ sampling atoms. The summation rule thus approximates the total potential energy according to

$$
V\left(\boldsymbol{q}^{h}\right)=\sum_{i=1}^{N} E_{i}\left(\boldsymbol{q}^{h}\right) \approx \widetilde{V}\left(\boldsymbol{q}^{h}\right)=\sum_{\alpha=1}^{N_{s}} w_{\alpha} E_{\alpha}\left(\boldsymbol{q}^{h}\right),
$$

where $w_{\alpha}$ is the weight of sampling atom $\alpha$ (roughly speaking, $w_{\alpha}$ defines the number of lattice sites represented by sampling atom $\alpha$ ). Typical distributions of repatoms and sampling atoms (which do not necessarily have to coincide) are shown in Fig. 1 for various types of summation rules.

In the nodal summation rule, repatoms also serve as sampling atoms. Cluster summation rules define the set of sampling atoms to be comprised of clusters of lattice sites surrounding all repatoms (Knap and Ortiz, 2001; Eidel and Stukowski, 2009). Quadrature summation rules include in the set of sampling atoms all repatoms as well as additional quadrature-type sampling atoms within elements (Gunzburger and Zhang, 2010). For all these summation rules, we compute weights $w_{\alpha}=\rho \Omega_{\alpha}$ from the cell volumes $\Omega_{\alpha}$ in a Voronoi tessellation based on the set of sampling atoms with $\rho$ being the effective atomic density. This choice yields the exact Hamiltonian in the atomistic limit and ensures consistency (Kochmann and Venturini, 2014). A new class of summation rules was introduced in (Amelang et al., 2015), which computes sampling atom weights such that spurious force artifacts vanish in the large element limit. This scheme allows for a fully-nonlocal QC formulation (without the need to differentiate between fully-atomistic and coarse-grained regions) and will be discussed below. For simplicity, we use the notation $\left(n_{C l}, n_{Q}\right)$ to denote a summation rule having $n_{C l}$ shells of atoms in clusters around repatoms and $n_{Q}$ quadrature sampling atoms per element. For example, the purely nodal summation rule is denoted by $(0,0)$, a 3 -shell cluster rule is $(3,0)$, and the four-point 


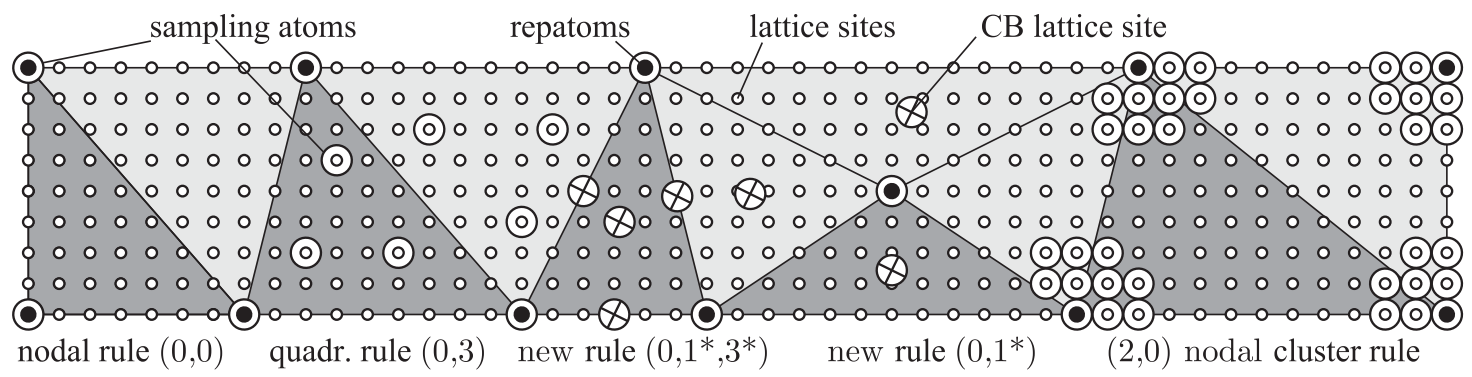

Figure 1: Schematic illustration of the QC discretization and different types of summation rules: the locations of sampling atoms shown in the dark elements correspond to quadrature and cluster summation rules as well as the new summation schemes of first and second order (CB denotes the Cauchy-Born rule applied to the neighborhood of sampling atom).

quadrature rule is abbreviated by $(0,4)$. In contrast to the original local/nonlocal QC formulation, see e.g. (Tadmor et al., 1996), we here only consider summation rules that include all repatoms in the set of sampling atoms to enable a seamless transition to full atomistic resolution upon mesh refinement. This way, full atomistic accuracy is recovered naturally when turning all lattice sites into repatoms (which are also sampling atoms in this limit) without the necessity of ad-hoc assumptions for the transition.

For the examples in subsequent sections we will chose potentials of the family of the Embedded Atom Method (EAM) (Daw and Baskes, 1984), which defines the potential energy $E_{i}$ of an atom $i$ by

$$
E_{i}(\boldsymbol{q})=\frac{1}{2} \sum_{j \neq i} \Phi\left(r_{i j}\right)+\mathcal{F}\left(\rho_{i}\right)
$$

with pair potential $\Phi\left(r_{i j}\right)$ depending on the distance $r_{i j}=\left|\boldsymbol{q}_{i}-\boldsymbol{q}_{j}\right|$ between atoms $i$ and $j$, and $\rho_{i}=\sum_{j=1}^{N} \phi\left(r_{i j}\right)$ the effective electron density. The force on repatom $k$ hence follows as

$$
\widetilde{\boldsymbol{F}}_{k}(\boldsymbol{x})=-\frac{\partial \widetilde{V}\left(\boldsymbol{q}^{h}\right)}{\partial \boldsymbol{x}_{k}}=-\sum_{\alpha=1}^{N_{s}} w_{\alpha} \sum_{j \in n_{I}(\alpha)} \frac{\partial E_{\alpha}\left(\boldsymbol{q}^{h}\right)}{\partial \boldsymbol{q}_{j}^{h}} N_{k}\left(\boldsymbol{X}_{j}\right),
$$

where we may truncate the inner sum to only those neighboring lattice sites $j$ contained within the sphere of interaction of sampling atom $\alpha$, abbreviated by $n_{I}(\alpha)$. As discussed above, the repatom force (4) contains residual/spurious force artifacts when applied to non-uniform QC meshes, unless $N_{s}=N$ (i.e., unless the summation is carried out over all atoms in the lattice which is prohibitively expensive from a computational standpoint), see e.g. (Eidel and Stukowski, 2009; Amelang et al., 2015). In Appendix A we derive the residual force on repatoms in a non-uniform 2D QC representation for instructive purposes. In all subsequent examples, we use the above framework to simulate the response of crystalline ensembles at zero temperature. In addition, we only consider quasistatic (low-strain-rate) phenomena so that inertial effects are negligible. Equilibration is performed using the inertial relaxation algorithm FIRE of Bitzek et al. (2006).

The QC method is primarily used to approximate long-range elastic fields. In the immediate vicinity of defects, full atomistic refinement turns our non-local QC scheme into lattice statics with no approximation errors or residual forces. Therefore, we concentrate on scenarios where the repatom spacing is larger than the atomistic limit. In our examples, we compare the following types of summation rules (Fig. 1 schematically shows the different rules):

- $(0,0)$ : a purely nodal summation rule,

- $\left(n_{C l}, 0\right)$ : nodal cluster summation rules with $n_{C l}$ shells of clusters,

- $\left(0, n_{Q}\right)$ : quadrature summation rules with $n_{Q}$ quadrature-type sampling atoms per element,

- $\left(0,1^{*}\right)$ : the new (first-order) summation rule introduced in (Amelang et al., 2015),

- $\left(0,1^{*}, 4^{*}\right)$ : the new (second-order) summation rule introduced in (Amelang et al., 2015), as well as its twodimensional counterpart $\left(0,1^{*}, 3^{*}\right)$. 
The new summation rule $\left(0,1^{*}\right)$ eliminates residual and spurious force artifacts in the limits of large elements and full atomistics. It was also shown to produce smaller energy approximation errors than any other summation rule, especially compared to those with comparable efficiency. In a nutshell, the new $\left(0,1^{*}\right)$ rule resembles a first-order quadrature rule: the set of sampling atoms includes all repatoms as well as one sampling atom per element whose neighborhood undergoes an affine deformation according to the Cauchy-Born rule. Weights are assigned such that in large elements all repatoms receive the same weight, viz. the number of lattice sites contained within an effective sphere of interaction around each sampling atom. The remaining lattice sites per element are represented by the weight of the Cauchy-Born sampling atom. In smaller elements, overlapping spheres of interaction are split symmetrically, approaching exact equal weights of $w_{\alpha}=1$ in the atomistic limit. Furthermore, we consider the second-order extension $\left(0,1^{*}, 4^{*}\right)$ with improved accuracy by accounting for the different energy levels of lattice sites located near element faces. To this end, we include additional sampling atoms, one per element face, whose weights are proportional to the size of the element faces and are chopped symmetrically with decreasing element sizes; for details see (Amelang et al., 2015). In summary, $\left(0,1^{*}\right)$ stands for a nodal summation rule with one additional sampling atom per element undergoing Cauchy-Born deformation, where as $\left(0,1^{*}, 4^{*}\right)$ denotes a nodal summation with one additional sampling atom per element, and one additional sampling per element face, all of which undergo Cauchy-Born deformations according to their interpolated local deformation gradients within elements and across adjacent elements, see also Appendix A. Note that, as mentioned previously, we exclude pure element quadrature rules from our analysis, since they do not transition to full atomistics without introducing some ad-hoc definition for the transition from local to non-local elements. Here, we focus only on fully-nonlocal QC schemes that seamlessly bridge across all element sizes.

\subsection{New first- and second-order summation rules}

As shown in (Amelang et al., 2015), the new summation rules of first and second order do not produce any residual or spurious force artifacts in affinely-deformed crystals discretized by sufficiently large QC elements because they satisfy two criteria: (i) all repatoms are assigned the same weights, and (ii) all remaining sampling atoms (i.e. those within elements and on element faces) feel only Cauchy-Born deformations of their local neighborhoods. In addition, the energy approximation error is minimized by introducing sampling atom locations and associated weights to optimally represent the total Hamiltonian by a limited number of sampling atoms. Sampling atoms on repatom sites (i.e. on nodes in the QC mesh) and sampling atoms on element faces represent the energies of all those lattice sites having neighbors that reach into adjacent elements (thus experiencing locally a different affine deformation). In contrast, sampling atoms within elements represent all those lattice sites well within elements and thus experiencing an affine deformation in the Cauchy-Born sense due to the chosen linear interpolation.

Fig. 2 illustrates the benefits of the new summation rules in a simple example. Shown is a QC representation having full atomistic resolution in the bottom left corner and coarse-graining away from that region in a randomized fashion. Next, we randomly displace all repatoms into a non-equilibrated deformed configuration. In this state, we compute the energy of each lattice site by considering its local atomic neighborhood as obtained from the QC interpolation. For a specific summation rule, each such energy is not considered exactly in the total (approximated) potential energy but is represented by the energy of a nearby sampling atom. Therefore, for each lattice site we compare its exact energy to that of the associated sampling atom. Fig. 2 shows the thus-obtained relative energy errors (each colored dot represents a lattice site). Of course, in the bottom left corner the error vanishes because full atomistics recovers the exact summation over all lattice sites. With coarse-graining away from the atomistic region, the error increases. We note that because the approximated and exact energies are computed from the same atomic positions, the shown energy errors are merely due to the summation rules and free from approximation errors incurred by the affine QC interpolation. Apparently, the new schemes choose sampling atoms and assign their weights such that the total energy error is considerably reduced compared to nodal and traditional quadrature schemes. Node-based cluster rules only improve over the shown purely nodal rule if the cluster size is sufficiently large so that clusters start to overlap, as explained also in (Eidel and Stukowski, 2009). Therefore, the addition of clusters around nodes does not noticeably alter the energy errors in sufficiently coarse-grained regions. While the first-order scheme effectively removes errors within elements, the second-order scheme also reduces the errors near element edges/faces, as expected. A related scenario of sharp mesh interfaces was studied in (Amelang et al., 2015).

As discussed above, energy approximation errors result in residual and spurious force artifacts. As an instructive example, Fig. 3 shows a three-dimensional QC mesh, again having full atomistic resolution near the bottom left corner 


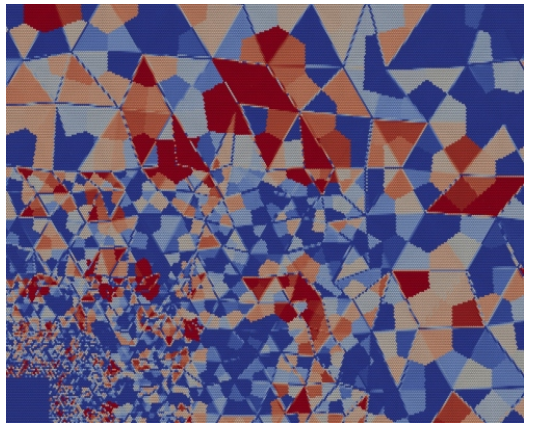

(a) nodal $(0,0)$

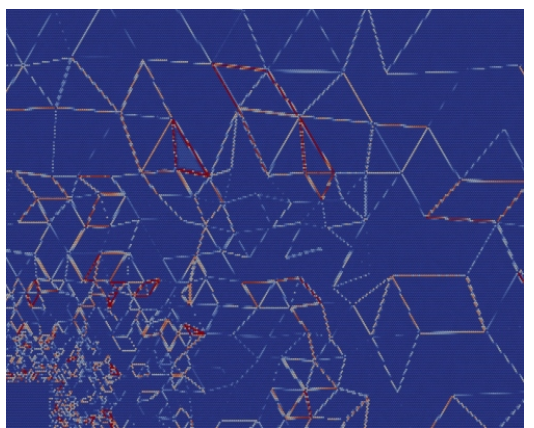

(d) new, first order $\left(0,1^{*}\right)$

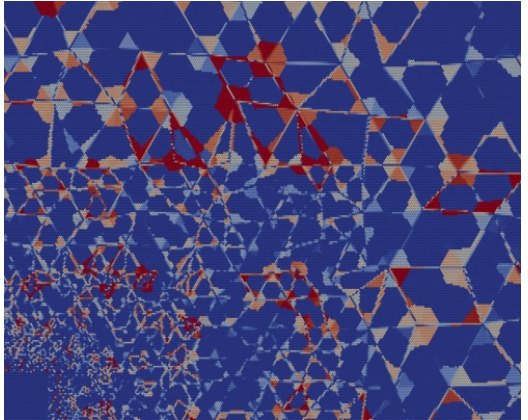

(b) quadrature $(0,1)$

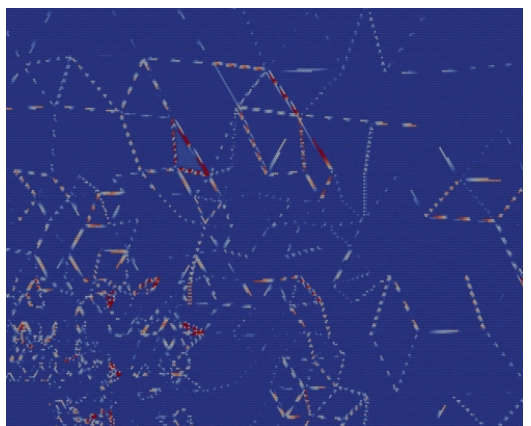

(e) new, second order $\left(0,1^{*}, 3^{*}\right)$

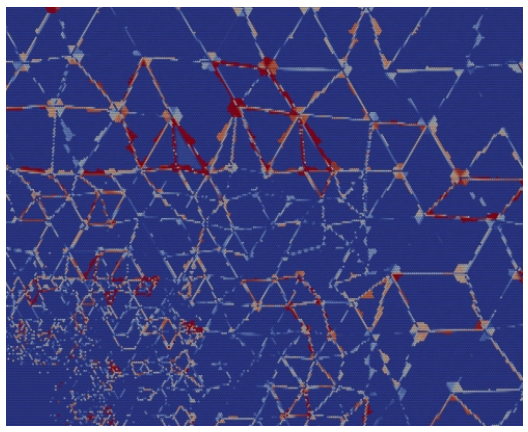

(c) quadrature $(0,3)$

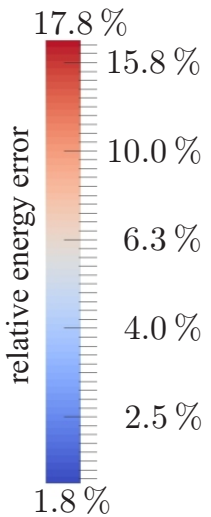

Figure 2: Energy approximation errors introduced by the summation rules in a non-uniform QC mesh with diffuse interfaces. After application of the affine QC interpolation, we compare the exact energy of each lattice site to that of the associated sampling atom for different summation rules.

and coarse-graining away from that region through sharp mesh interfaces. The arrows illustrate the residual forces appearing in the undeformed initial configuration (in which no such net forces exist if the sample was studied by full atomistics; i.e., these forces are indeed modeling artifacts). Here, the addition of clusters indeed reduces the magnitude of the force artifacts significantly below those observed for the purely nodal summation rule (compare cases (a) and (d)). In contrast, the new summation rules remove all force artifacts in the coarse-grained regions except for a small region (where elements are sufficiently small so that nodal spheres of interaction start to overlap). Similarly to the classical local/nonlocal QC formulation of (Tadmor et al., 1996), force artifacts only appear near the interface between fully atomistic and coarse-grained regions with the main difference that the formulation does not require the notion of such an interface (there is no conceptual difference between atomistic and coarse-grained regions anymore).

\subsection{Free surfaces and the QC method}

The relaxation of free surfaces leads to a local rearrangement of atomic positions in the crystal lattice due to a loss of symmetry in atomic neighborhoods near the surface, see e.g. (Rous, 1995; Inglesfield, 1995) for comprehensive overviews. Depending on the atomic structure and interactions, surface relaxation can be as simple as changing only the spacing between atomic layers near the surface, but it can also involve a complex surface reconstruction (oftentimes seen in transition metals). Here, we restrict our attention to non-reconstructing surfaces of metals which result in a change of the spacing between atomic layers close to the free surface, see Fig. 4a. That is, atomic positions are relaxed normal to the surface. For most metals, this phenomenon affects primarily the distance between the first few atomic layers, although it can extend deeper depending on the material. Also, most metals exhibit a contraction of the atomic layers; only group 2 and 3 metals (for example, beryllium and aluminum) have been shown to produce an expansion of the surface layer spacing. To what extend atomic layers are affected by the surface relaxation depends on the effective range of atomic interactions (in the following denoted by $r$ ), which in simulations is related to the cut-off radius of the potential (the effective interaction distances may be shorter due to long tails of the potentials). 


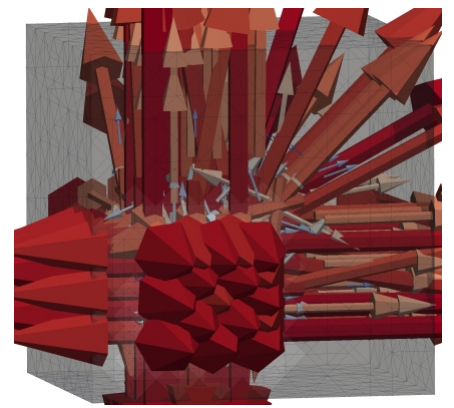

(a) nodal $(0,0)$

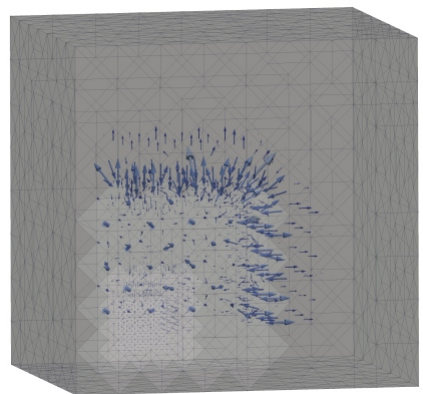

(d) cluster $(3,0)$

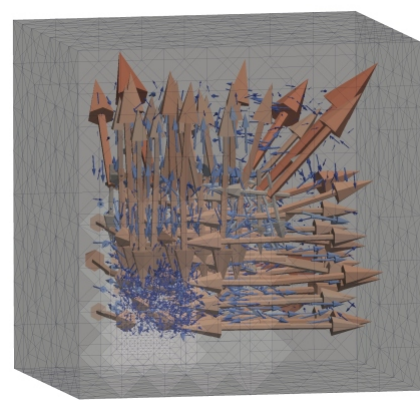

(b) quadrature $(0,1)$

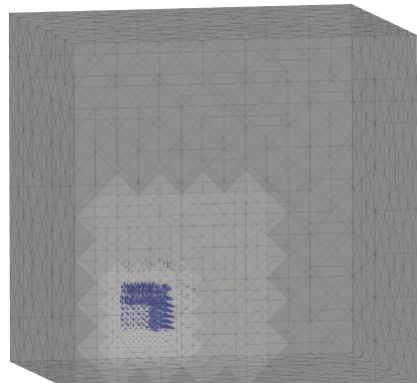

(e) new, first order $\left(0,1^{*}\right)$

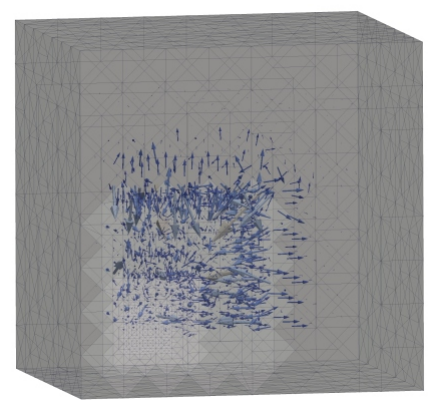

(c) quadrature $(0,4)$

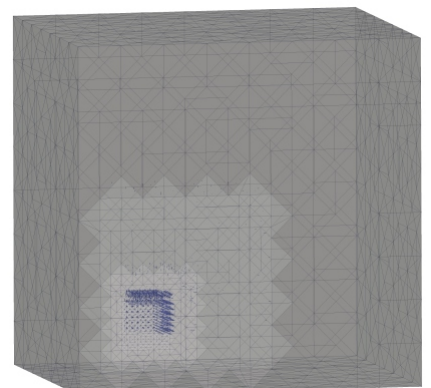

(f) new, second order $\left(0,1^{*}, 4^{*}\right)$

Figure 3: Residual forces in a non-uniform QC mesh with sharp interfaces for various summation rules in three dimensions (including the new summation rules of first and second order). The color code reflects residual force magnitudes (in arbitrary units on a logarithmic scale).

In the following, we tacitly assume that the chosen interatomic potential can capture surface effects with sufficient accuracy. In this case, a full atomistic simulation can easily determine the relaxed surface configuration by equilibrating the atomistic ensemble in the presence of free surfaces. Depending on the material and the surface orientation, this geometric relaxation effect might be rather small; e.g., changes in atomic layer spacings below planar surfaces in pure $\mathrm{Cu}$ range between $1-10 \%$ for the first layer (for different surface orientations) and decay quickly with increasing depth (Rous, 1995). However, the presence of a surface significantly affects the mechanics of those layers nearest to the surface. Leaving aside the various inelastic surface-dominated mechanisms (e.g., the attraction of moving dislocations, single-arm dislocation sources, or crack initiation), the elastic performance of the crystal near the surface gives rise to size effects in the elastic moduli.

When using the QC method to model small-scale structures, the coarse-graining of crystalline regions near free surfaces creates problems. Of course, the chosen interpolation within elements prevents individual atomic layers from adjusting their equilibrium spacing near the surface (but, as argued above, this geometric effect is rather small). However, a considerable error arises from the introduction of summation rules if all inner-element lattice sites are represented by sampling atoms on or near the surface (such as in nodal or node-based cluster summation rules), or if all surface-atoms are represented by inner-element sampling sites whose energies remain unaffected by the surface (such as in the classical local QC formulation using the Cauchy-Born rule for all coarse-grained elements). Fig. 4b visualizes the distribution of sampling atoms for both a traditional nodal cluster summation rule with tesselated weights and the new second-order summation rule within elements adjacent to the free surface. The new scheme achieves a highly-accurate representation of surface-nearest lattice sites by sampling atoms on the surface. Alternatively, many previous QC simulations have made use of full atomistic refinement near the surface, see (Fang and Yang, 2014) for a recent example. While this approach is most accurate, it also incurs tremendous computational expenses that become prohibitive when modeling large, above-nanoscale systems.

The new second-order summation rule discussed above avoids this problem through sampling atoms on all element faces (or edges in 2D). Their weights are chosen such that they automatically represent only those face-nearest lattice sites whose energies deviate from that in the bulk. Of course, this accounts for free surfaces only in an average sense (all surface-nearest atoms are approximated by the same energy per atom). Yet, the scheme ensures that (i) the total 
(a)

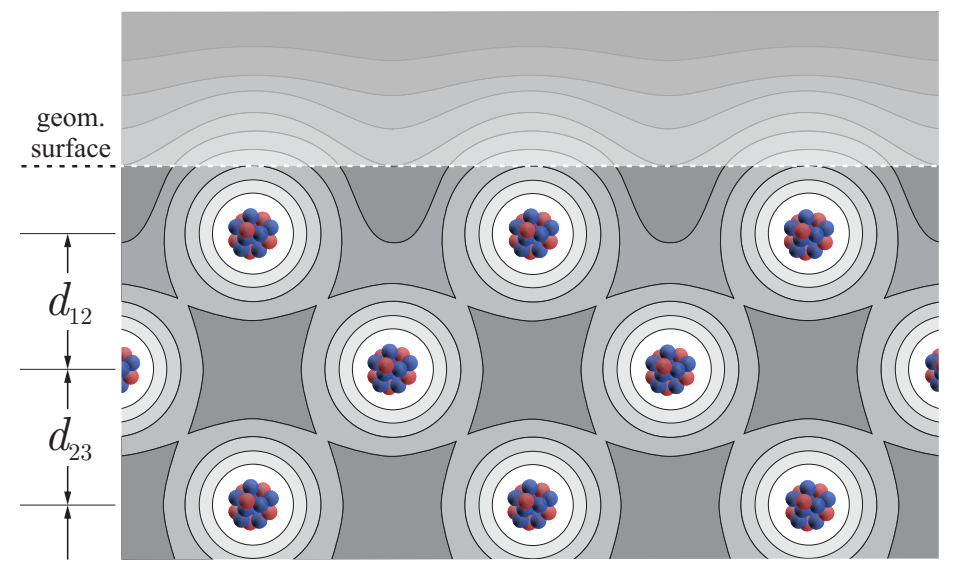

(b) (light) regions represented by sampling atoms in the bulk

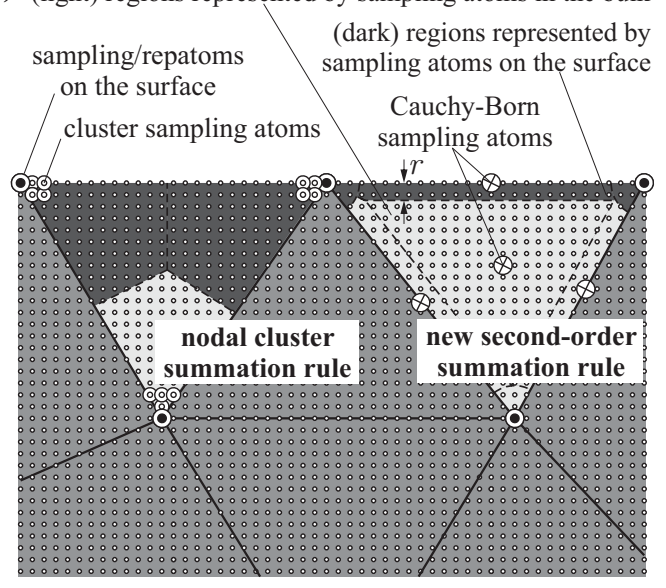

Figure 4: (a) Schematic view of the structure of a free surface in a metallic crystal: positions of atomic cores and the corresponding electron density distribution with the characteristic spacings of atomic layers below the geometric surface ( $d_{i j}$ deviate from the lattice spacing $a_{0}$ near the surface). (b) Visualization of a classical nodal cluster summation rule and the new second-order summation rule along with their repatoms and sampling atoms in simplicial elements near the surface (dark regions represent those lattice sites represented by sampling atoms on or near the surface for the example of an effective atomic interaction distance of $r=1.5 a_{0}$ ).

potential energy is approximated fairly well by accounting for both surface and bulk atoms, and (ii) only minimal force artifacts arise from free surface in non-uniform QC meshes. As we will show in the following examples, the latter is an important observation because e.g. a purely nodal summation rule can lead to large spurious deformation of the free surfaces in response to residual force artifacts. Also, residual forces can no longer be identified as such since the undeformed configuration of a non-uniform QC mesh now exhibits both non-physical force artifacts and real relaxational forces near the surface, the distinction between which is impossible without running a full MD simulation for comparison.

\section{Numerical examples}

The above discussions have shown that the fully-nonlocal energy-based QC methodology along with the new second-order summation rule of (Amelang et al., 2015) seems well suited to capture surface effects in nanoscale structures; however, the technique has never been applied to investigate nanoscale size effects. In the following, we report a series computational studies that probe elastic size effects and inelastic surface effects in small-scale structures by the QC approach outline above.

\subsection{Calculation of surface energies}

The calculation of surface energies by molecular statics (or dynamics) is straight-forward and can provide reliable data when suitable interatomic potentials are being used, see e.g. (Todd and Lynden-Bell, 1993) for a representative study. In contrast, coarse-grained atomistic simulations commonly fail to accurately capture such surface characteristics due to (i) spurious force artifacts near the surface which lead to an incorrect deformation of the atomic layers near the surface, and (ii) the errors made by approximating the potential energy through summation rules. Consequently, the total energy of QC samples with free surfaces may contain significant errors. Therefore, surface energies are a good indicator for the accuracy of our coarse-grained atomistic description.

As an example, we compute the surface energy of (100) pure single-crystalline $\mathrm{Cu}$ modeled by the extended Finnis-Sinclair potential of Dai et al. (2006) and by the potential of Mishin et al. (2001) at zero temperature. To this end, we construct a square slab with planar top and bottom (100) surfaces and with periodic boundary conditions imposed on the lateral faces, see Fig. 5. We equilibrate the nano-crystal to identify all relaxed atomic positions (i) by molecular statics and (ii) by the QC method with the various summation rules discussed in previous sections. We compute the energy of the relaxed sample and contrast it with the energy of the same number of atoms in an infinite 


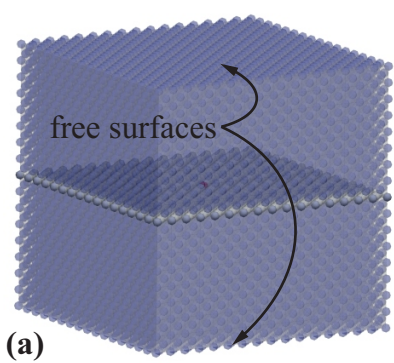

(a)
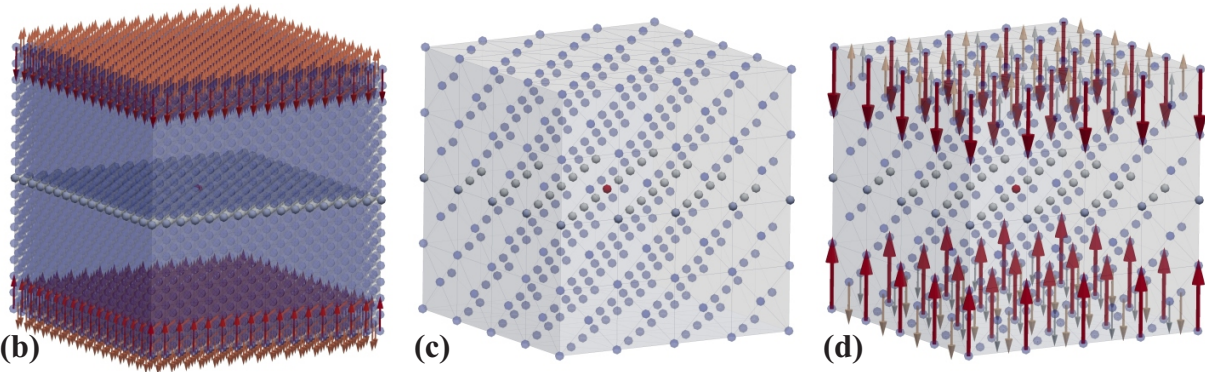

Figure 5: Schematic view of the model used to determine surface energies: (a) full atomistic model (with free surfaces at the top and bottom and periodic boundary conditions otherwise; the center plane of atoms is restrained to suppress rigid body motion), (b) forces appearing in the initial configuration and resulting in surface relaxation, (c) the same cube modeled by a coarse-grained QC repatom distribution with free surfaces at top and bottom, and (d) forces appearing in the initial QC configuration, comprising both surface forces and, in principle, erroneous force artifacts.
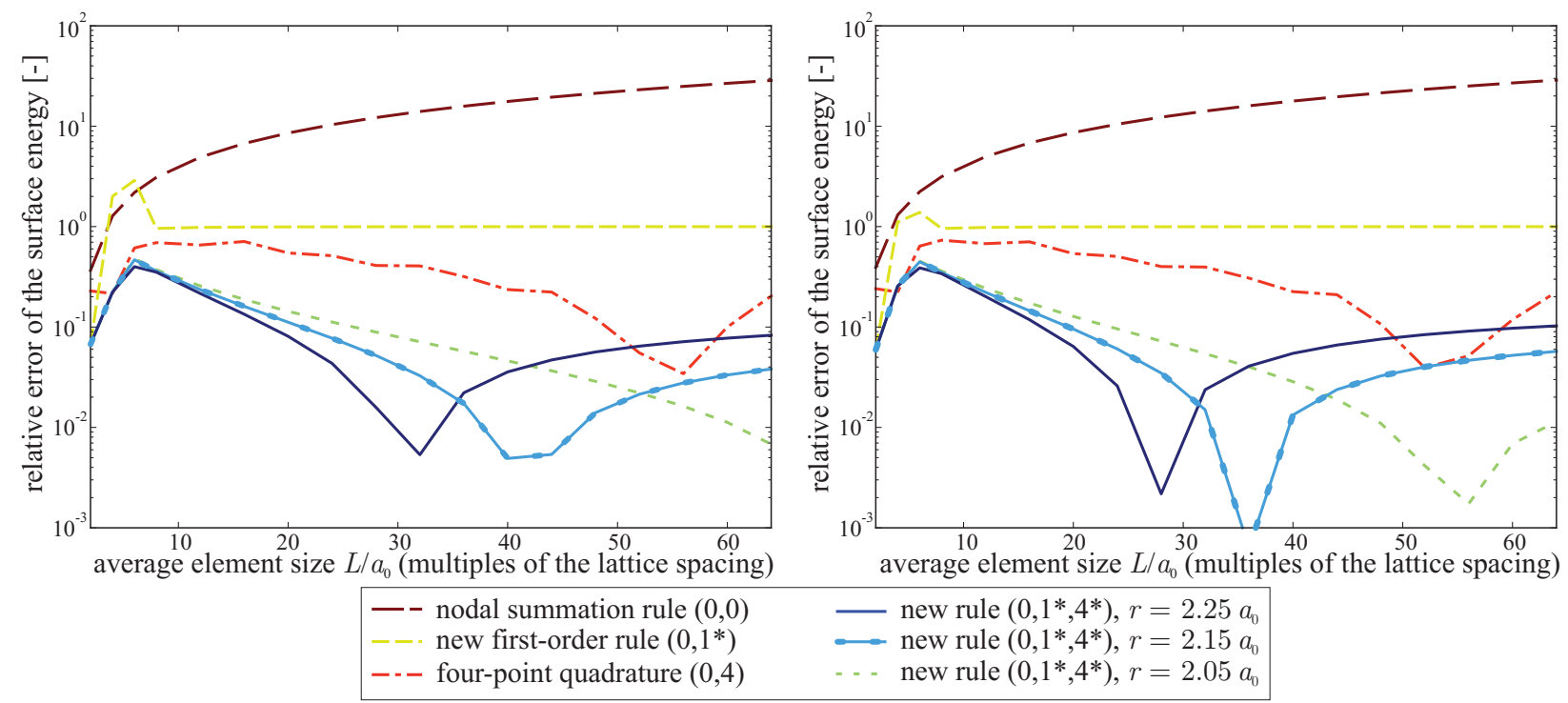

Figure 6: Relative error of the computed surface energy vs. QC element size for single-crystalline (100) Cu modeled by the potentials of (a) Mishin et al. (2001) and (b) Dai et al. (2006) for various summation rules, including the new second-order rule with different effective interaction radii $r$.

single crystal. The excess energy divided by the total surface area provides the surface energy. In addition to the choice of the summation rule, results generally also depend on the mesh size and quality.

Fig. 6 shows the relative error of the computed surface energy as a function of the average element size in the QC representation for six different summation rules, including the new schemes of first and second order. The new rule of second order shows considerably lower errors than the other schemes across all element sizes tested, especially in the limit of large elements where the error is as low as a few percent (while comparable other schemes exhibit errors on the order of $100 \%$ and above). Of course, the error depends essentially on the choice of the effective interaction radius of the chosen potential (which does not have to agree with the potential cut-off due to long shallow tails of the most common potentials). Therefore, Fig. 7 illustrates the error as a function of the element size and of the effective interaction radius $r$, showing optimal $r$-values near $r=2 a_{0}$ in the limit of large elements with a major deviation only apparent for element sizes between $10-20 a_{0}$. This allows us to define the optimal values for the effective interaction radius $r$ (to be used in the following). This QC scheme has been tested with various other potentials not shown here for brevity, and the same trends have been observed.

It is important to keep in mind that the potential cut-off radius enters our QC model in two very distinct ways, only one of which is our focus here. On the one hand, the cut-off radius is essential for the calculation of interatomic forces as in every atomistic model. This actual cut-off radius comes with a potential and is not modified here (changing this 


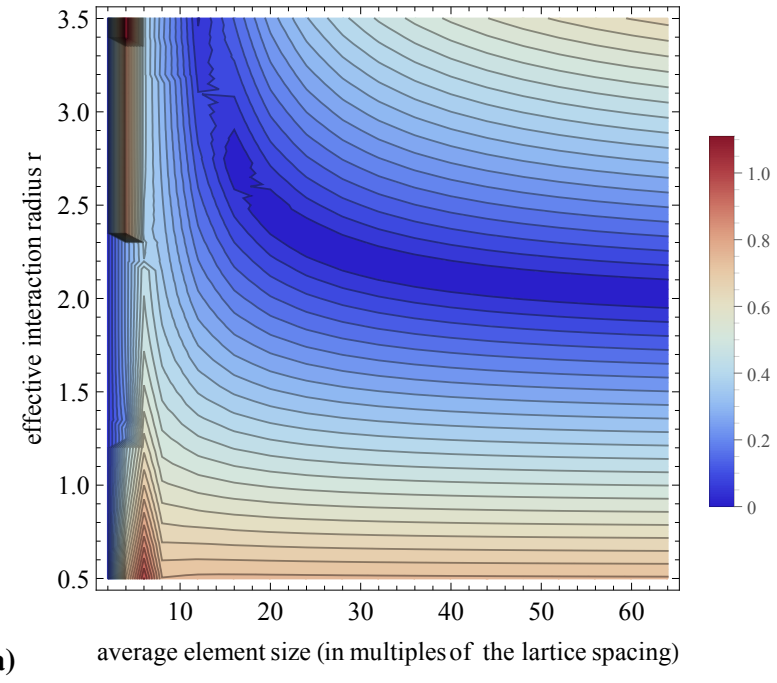

(a)

Figure 7: Contour plot of the relative error of the surface energy as a function of the effective interaction radius $r$ and the average element size $L / a_{0}$ (in multiples of the atomic spacing) for the potentials of (a) Mishin et al. (2001) and (b) Dai et al. (2006), showing convergence for large elements.

cut-off radius may lead to significant changes in material properties). On the other hand, our new first- and secondorder summation rules define the weights of sampling atoms in dependence of an effective cutoff radius (which is a good measure for how many atoms in the vicinity of e.g. an element node are best represented by the lattice site located at that node rather than those lattice sites within elements). This is different from all previous summation rules which defined sampling/repatom weights based on consistency or based on geometric arguments. Here, the sampling atom weights depend on the effective interaction radius $r$, and the consequences of changing $r$ for weight calculations (not for interatomic force calculations) is shown in Fig. 7.

\subsection{Elastic modulus of a nano-rod}

In order to assess the impact of free surfaces on the elastic response of nanoscale structures, we simulate the uniaxial extension of a nano-rod with square cross-section (while circular cross-sections are more realistic and common in nano-wire experiments, the flat surfaces admit a cleaner investigation without complicating geometric effects). We deliberately consider only elastic deformation here to avoid the formation of microstructure which quickly leads to a randomized response, so that results cannot be compared directly to full atomistics, see also the discussion in (Amelang et al., 2015). Defects are considered in Section 3.4. For various summation rules and mesh sizes, we compute the load-displacement curve for the uniaxial extension of differently-sized whiskers whose length is six times the crosssectional side length (the latter is varied to demonstrate size effects). From the resulting stress-strain relations, we extract the effective, size-dependent (structural) Young's modulus (linearized about the undeformed state) to quantify the impact of free surfaces on the elastic response, cf. (Miller and Shenoy, 2000).

Fig. 8 illustrates the stress-strain response of the rod for six different summation rules and for three different mesh sizes of the QC representation (a side length of $L=4 a_{0}$ implies that element side lengths on the surface amount to four lattice spacings). Obviously, for small element sizes (Fig. 8a), the impact of the various summation rules is small and the stress-strain curves obtained from QC agree well with that obtained from lattice statics (which is also included in each of the three plots). As the element size increases, the summation rules gain importance. The new second-order summation rule is by far the most accurate, showing a response that is almost identical to the exact atomistic one. Most importantly, this is true for arbitrary coarsening ratios, whereas the errors produced by all other summation rules grow considerably with element size (the next best rule being four-point quadrature in this comparison).

Fig. 9 shows the deformed nano-rods for different summation rules and for $L=16 a_{0}$ (the rod has cross-sectional side lengths of $11.6 \mathrm{~nm}$ ). The graphics show both the exact solution (light) and the QC solution (dark blue), thus indicating the errors in the deformed shape of the rod. Large errors arise from the nodal summation rule, where 

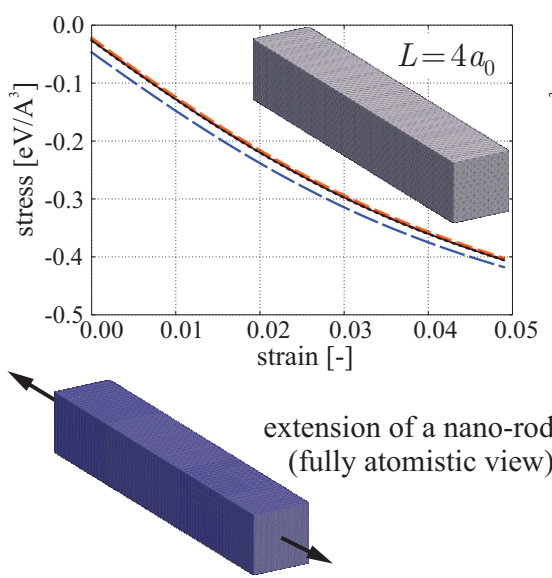
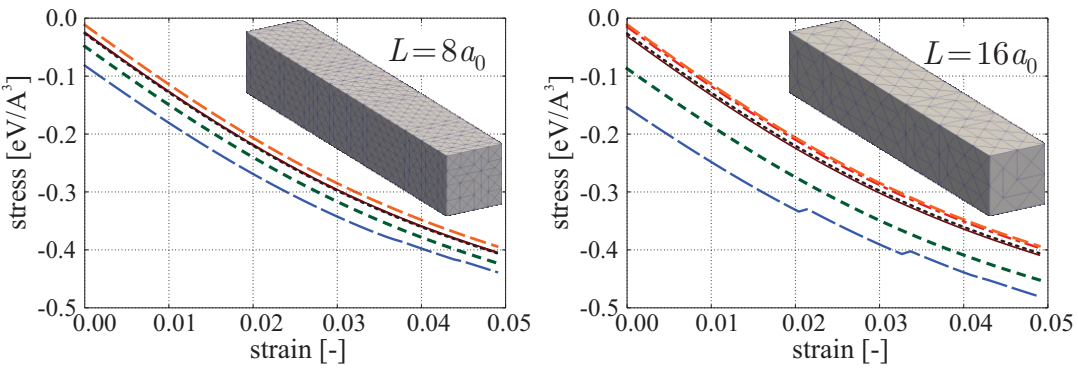

$$
\begin{array}{|cl|}
\hline-\cdots-\text { full atomistics } & --- \text { four-point quadrature }(0,4) \\
-- \text { nodal summation rule }(0,0) & --- \text { new first-order rule }\left(0,1^{*}\right) \\
--- \text { cluster summation rule }(3,0) & - \text { new second-order rule }\left(0,1^{*}, 4^{*}\right) \\
\hline
\end{array}
$$

Figure 8: Stress-strain response of nano-rods of different sizes modeled by the QC method with five different summation rules and three different average element sizes $L$. The rod is stretched uniaxially with unconstrained lateral surfaces; the full atomistic solution is included for comparison. (Note that at end effects are responsible for the observed initial stress at zero applied average strain.)

spurious force artifacts lead to a severely-deformed surface with artificial roughness. Again, the new second-order summation rule reproduces the deformed configuration in convincing agreement with the exact solution.

Evaluating the stress-strain response about the undeformed state yields the effective (structural) Young's modulus; i.e., we compute the slope of stress-strain curves such as those in Fig. 8 at zero strain. Results are plotted in Fig. 10a vs. the cross-section of the single-crystalline $\mathrm{Cu}$ nano-rod. In the limit of large diameters, the uniaxial modulus in the (100) direction agrees well with the expected value for a linear elastic material with cubic symmetry. The elastic constants can be determined from the interatomic potential via the Cauchy-Born rule, see e.g. (Tadmor et al., 1996), which yields $C_{11}=168.4 \mathrm{GPa}, C_{12}=121.4 \mathrm{GPa}$, and $C_{44}=75.4 \mathrm{GPa}$ in agreement with experiments (Kittel, 1996). Young's modulus for uniaxial tension-compression then follows as

$$
E=\frac{\left(C_{11}-C_{12}\right)\left(C_{11}+2 C_{12}\right)}{C_{11}+C_{12}}=66.69 \mathrm{GPa}=0.417 \mathrm{eV} / \mathrm{A}^{3}
$$

in agreement with (Simmons and Wang, 1971). As becomes apparent from Fig. 10a, this value is approached correctly in the limit of large rod cross-sections. Fig. 10b illustrate the error introduced by the various summation rules, which confirms the general trends observed before with the new second-order summation rule showing errors of less than $2.5 \%$ throughout.

(a) full atomistics

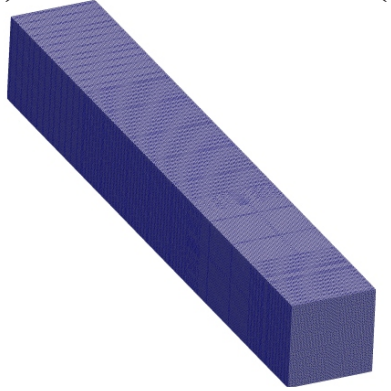

(b) nodal rule $(0,0)$

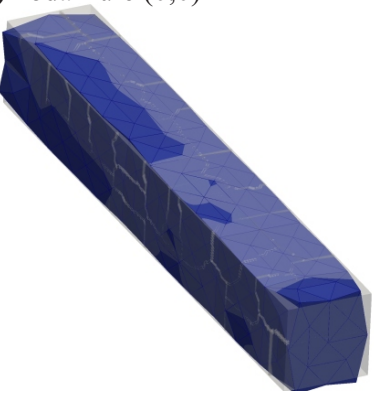

(c) quadrature rule $(0,4)$

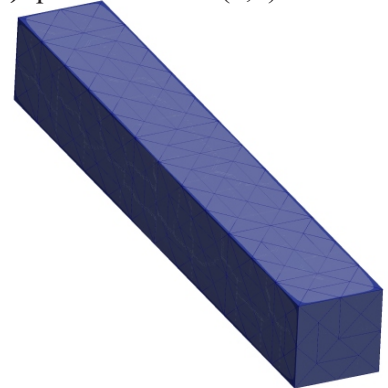

(d) new rule $(0,1 *, 4 *)$

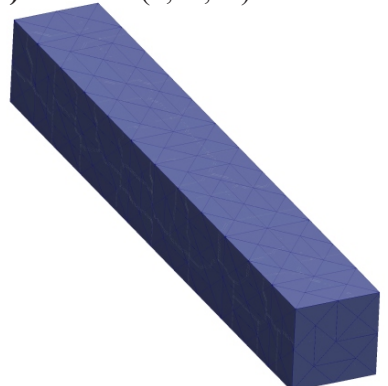

Figure 9: Deformed Cu nano-rods under uniaxial extension: (a) full atomistic solution, and (b)-(d) approximate QC solutions (the dark regions highlight deviations from the exact atomistic result). Particularly the nodal rule shows evidence of large errors due to force artifacts. 

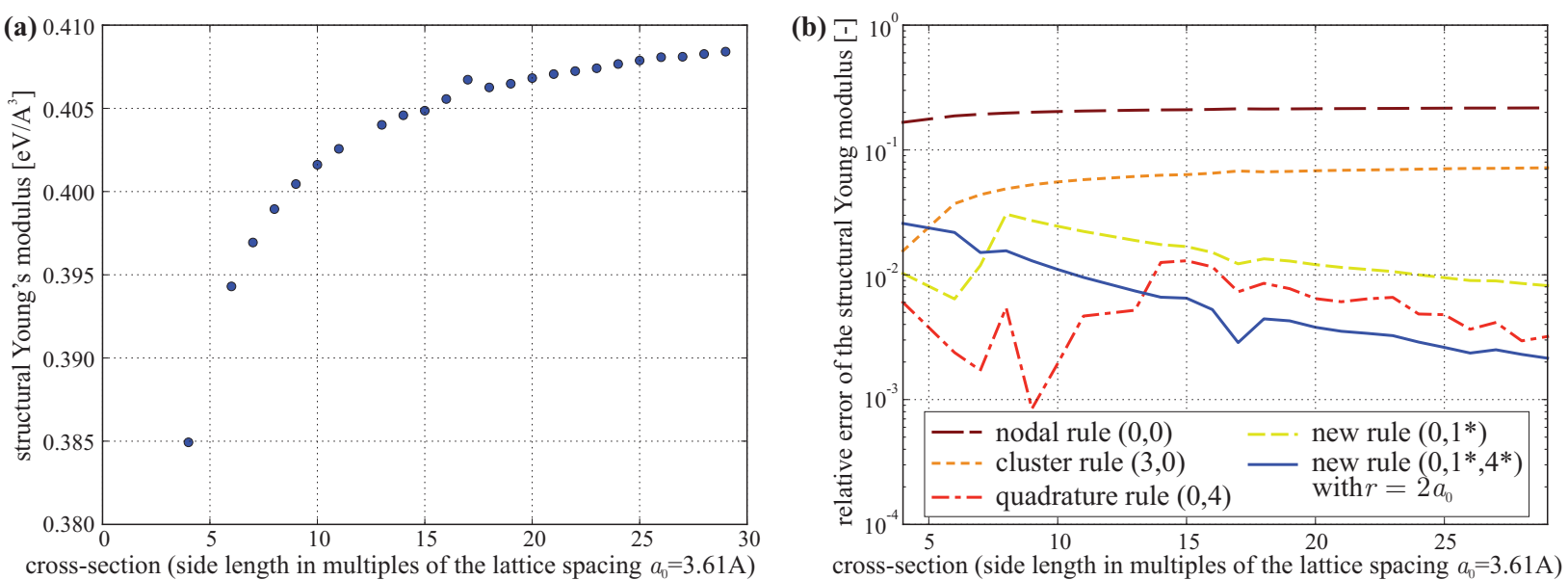

Figure 10: Size effects of the elastic modulus of a nano-rod: (a) elastic modulus vs. side length of the square cross-section of the nano-rod under uniaxial tension-compression; (b) error of the elastic modulus obtained form a QC representation of the nano-rod using various summation rules.

\subsection{Relaxation of a nano-cube}

So far, we have modeled surfaces by coarse-grained atomistics using static meshes. One of the benefits of the fullynonlocal QC method used in this study is its adaptive character which allows us to locally refine the mesh down to full atomistic resolution where required. The mesh refinement algorithm is based on the criterion proposed in (Knap and Ortiz, 2001): for each element in the QC mesh the second invariant of the (constant) deformation gradient is computed and compared to a tolerance level. If at any iteration step the invariant surpasses that refinement tolerance, then the element is selected for refinement. Element refinement is achieved by a modified bisection algorithm that splits tetrahedral elements into half by splitting the longest edge. Elements are refined in arbitrary order, and after each round of refinement the QC model is equilibrated; refinement continues until a stable equilibrium is reached.

We quasistatically relax the initial configuration of a nano-cube of side length $11.52 \mathrm{~nm}$ whose edges align with the (100) directions of the $\mathrm{Cu}$ single crystal modeled by the extended Finnis-Sinclair potential of (Dai et al., 2006). Surface relaxation leads to a deformation of the cube without any applied loads, and the aforementioned refinement algorithm is applied to achieve higher accuracy near the surface of the cube. Fig. 11a shows the resulting total number of sampling atoms in the QC representation of the cube compared to the total number of lattice sites in the cube. For large values of the refinement tolerance (effectively suppressing refinement overall), all QC models have the same underlying mesh and thus the number of sampling atoms e.g. for a tolerance of 10 merely indicates the different numbers of sampling atoms resulting from the different summation rules. Not considering the initial simulation setup, the computational costs of a summation rule depend on the number of energy/force evaluations and are thus proportional to the number of sampling atoms. Apparently, the efficiency of the new scheme $\left(0,1^{*}, 4^{*}\right)$ is comparable to that of quadrature rule $(0,4)$, and it is considerably more efficient than the traditional cluster rule $(3,0)$. For small values of the refinement tolerance, all simulations approach full atomistic resolution.

The total energy of the relaxed cube contains essential contributions from atoms on and near the surfaces. Fig. 11b shows the total error introduced by five different summation rules (the new second-order rule is included with two different choices of the effective interaction distance $r$ ). Of course, one cannot expect that large coarsening ratios (i.e., high refinement tolerances) reproduce the energy correctly, especially in such a small cube where the relaxation near edges and vertices deviates from the planar geometry studied in Section 3.1. Results show that for large element sizes (high refinement tolerance) the error produced by the new summation rules is orders of magnitude less than that of the nodal and cluster rules, and comparable to four-point quadrature. Even with refinement the new second-order rule overall performs best and offers a significant improvement.

\subsection{Plate with a cylindrical hole}

Finally, let us use the same QC methodology to model the failure of a thin $\mathrm{Cu}$ plate with an initially cylindrical hole, loaded in uniaxial extension in a (100) direction. The single-crystalline plate, shown in Fig. 12, has extensions 


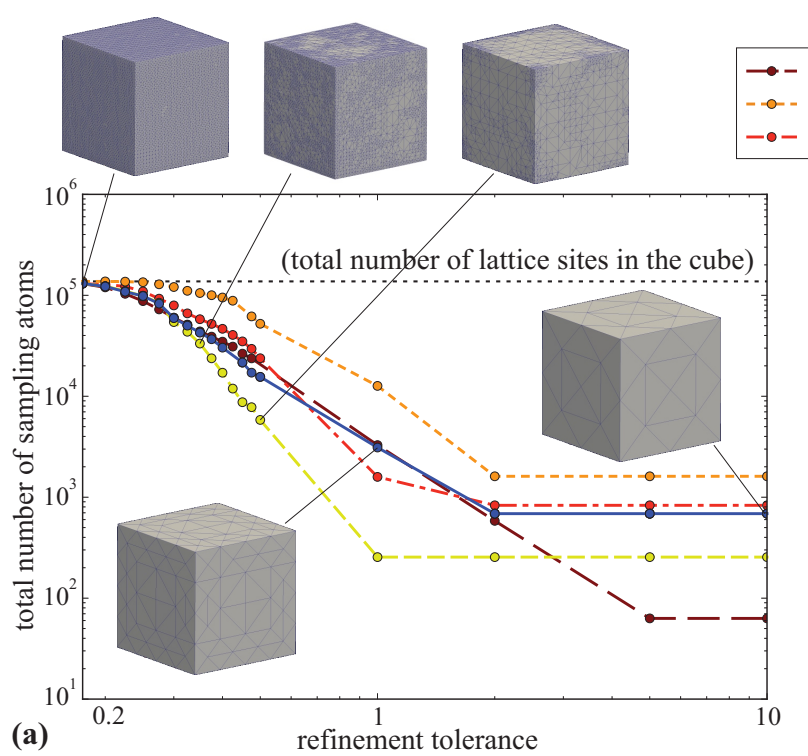

$\begin{array}{lll}\rightarrow-\text { nodal summation rule }(0,0) & -0 & \text { new first-order rule }\left(0,1^{*}\right) \\ -- \text { - cluster summation rule }(3,0) & \rightarrow & \text { new rule }\left(0,1^{*}, 4^{*}\right), r=2.4 a_{0} \\ -- \text { - four-point quadrature }(0,4) & -\bullet- & \text { new rule }\left(0,1^{*}, 4^{*}\right), r=1.8 a_{0}\end{array}$

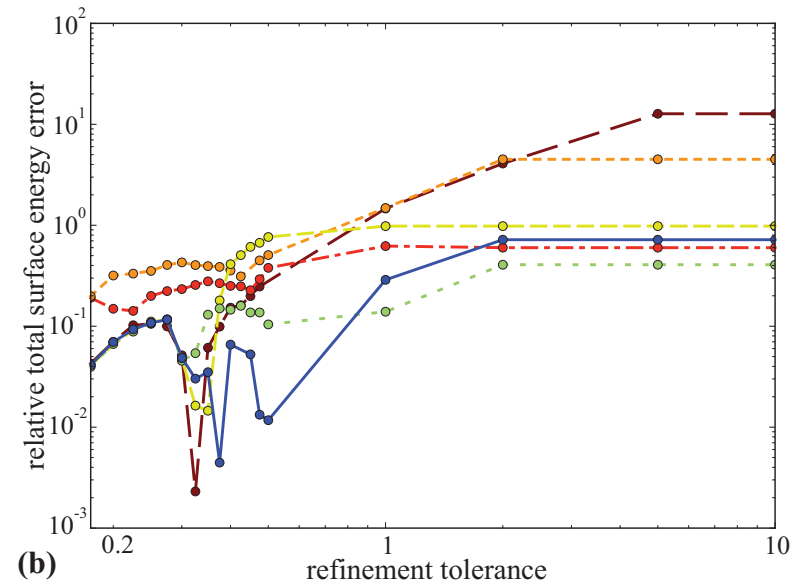

Figure 11: Relaxation of a free-standing nano-cube with adaptive mesh refinement: (a) total number of sampling atoms in the QC representation of the cube resulting from different values of the refinement tolerance (graphics show the cube after refinement for different refinement tolerance levels); (b) error of the approximated total surface energy of the cube vs. refinement tolerance for various summation rules.

of approximately $92.4 \mathrm{~nm} \times 92.4 \mathrm{~nm} \times 11.6 \mathrm{~nm}$ with a cylindrical hole at its center whose diameter we vary from about $3 \mathrm{~nm}$ to $6 \mathrm{~nm}$. The region immediately surrounding the hole is modeled by full atomistics while the remainder of the plate is efficiently coarse-grained (of course, this is only a representative example - the general technique is applicable to, in principle, arbitrary system sizes).

We model the same scenario with a continuum description using the finite element method to obtain an approximate solution of the stress and strain distribution inside the plate with a cylindrical hole of the same dimensions.
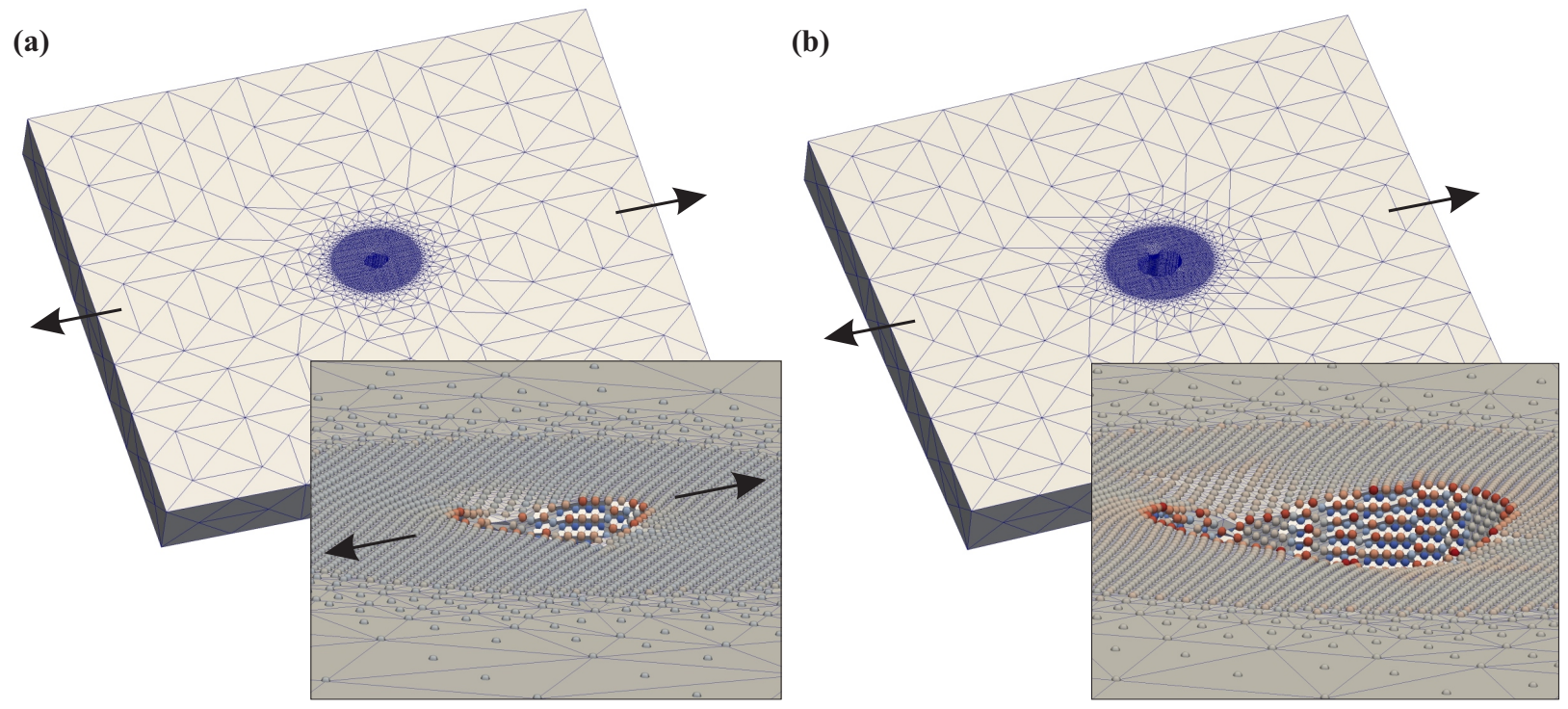

Figure 12: QC models of a thin plate with a cylindrical hole of (a) $3 \mathrm{~nm}$ and (b) $6 \mathrm{~nm}$ in diameter with zoomed graphics showing the deformation of the surface-nearest atomic sites in the vicinity of the hole at approximately (a) $2.8 \%$ and (b) $3.3 \%$ uniaxial strain (immediately before failure). Near the hole is full atomistic resolution with coarse-graining away from the center (arrows indicate the tensile loading direction). 

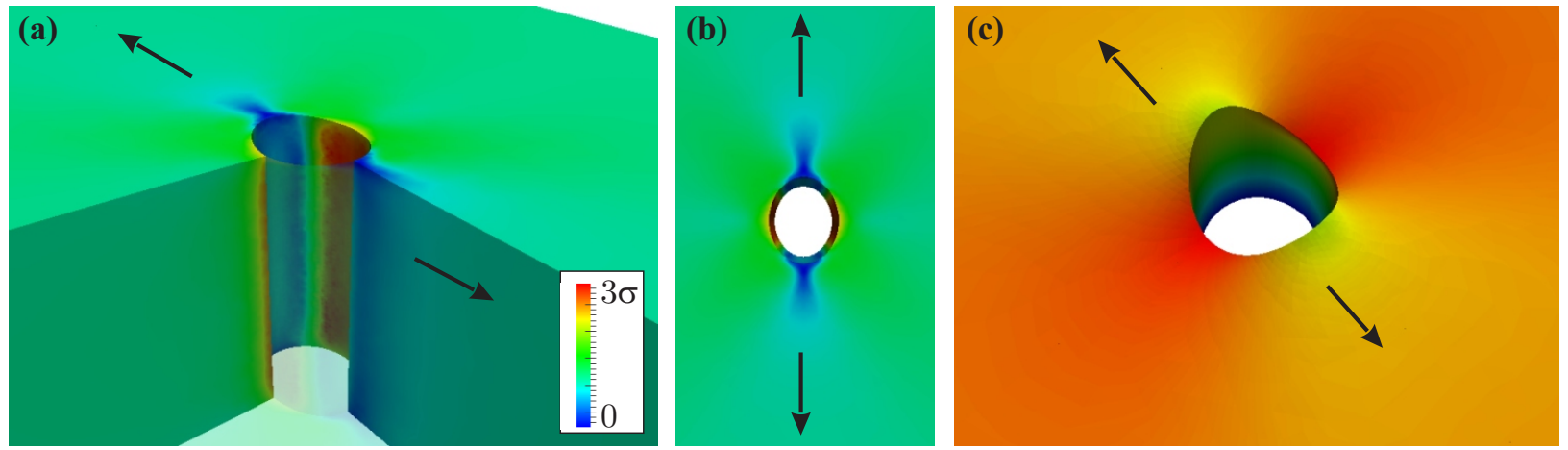

Figure 13: Finite element results for uniaxial loading at 2.4\% strain, demonstrating the von Mises stress distribution (a) in the vicinity of the hole in three dimensions (including a cut through the sample) and (b) in a top view. The legend shows the stress normalized by the applied remote loading. Stress concentrations appear where microstructure is observed to form in Fig. 14; (c) illustrates the surface displacements near the hole (with out-of-plane displacements magnified by a factor of 40 for clarity and colors on the same color legend normalized by the maximum displacement).

In order to mimic the atomistic scenario, we use a St. Venant-Kirchhoff hyperelastic energy density whose modulus tensor components $\mathbb{C}_{i j k l}$ obey cubic symmetry with the stiffness constants obtained in Section 3.2 from the interatomic potential of (Dai et al., 2006) (which is used in the QC simulations). Of course, the continuum description is independent of the problem size and the ratio of the hole diameter to the plate thickness plays only a minor role for the chosen geometry. Fig. 13 summarizes results obtained from the continuum study, including the out-of-plane deformation of the surface near the hole (matching quite well the atomistic/QC results of Fig. 12) as well as the von Mises-stress distribution in the vicinity of the cylindrical hole with large stress concentrations at the hole on the axis perpendicular to the loading direction, as can be expected theoretically.

When loading the coarse-grained atomistic simulations of Fig. 12 beyond the elastic limit, microstructural defects form near those locations of highest von Mises stress. Fig. 14 shows the formation of incipient dislocation loops spreading from the cylindrical hole and from the surface of the plate before the plate fails through cleavage (at zero temperature the dominant mode is brittle failure). Quite clearly, the surface plays an important role by affecting the stress state near the surface and by nucleating defects from and near the surface. The result is a size effect: while the plate with a $3 \mathrm{~nm}$ hole fails near $2.8 \%$ applied strain, the $6 \mathrm{~nm}$ hole fails not until $3.3 \%$ applied strain.

Of course, the simulation of the hole and its immediate vicinity could easily have been carried out using MD due to the small hole diameter. However, the long-range elastic fields resulting from the hole and, in particular, due to microstructural defect formation require an appropriate representation far away from the cylindrical hole. Here, the QC methodology is a powerful tool that enables the modeling of, in principle, arbitrarily large systems containing atomistic features through coarse-graining; and unlike all previous schemes, free surfaces can be accounted for successfully by the presented new summation rules of second order.

\section{Conclusions}

We have applied the fully-nonlocal energy-based quasicontinuum method to nanoscale structures including nanoparticles, nano-rods, and plates with nano-sized holes to demonstrate the impact of free surfaces on the elastic and plastic material response. The second-order optimal summation rule used in the QC method was shown to accurately and efficiently capture the influence of free surfaces in a few representative examples. Going beyond all previous QC techniques, the present multiscale modeling approach enables us to investigate the mechanics and physics of nanoscale systems efficiently through coarse-grained atomistics without the need to introduce ad-hoc corrections for free surfaces. Instead, the chosen approach naturally accounts for surface effects and enables the study of size effects in nanoscale metals.

We would like to point out that most problems discussed here could alternatively be resolved by having full atomistic resolution across the entire surface, and this strategy has indeed been pursued in the scientific literature. However, full atomistic resolution on all surfaces implies high computational expenses, which severely limits the domain size to be modeled. If interesting phenomena can be expected to happen below the surface (e.g., defect 
(a)

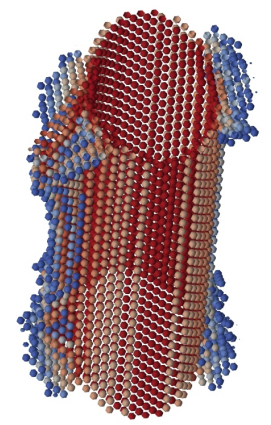

(b)

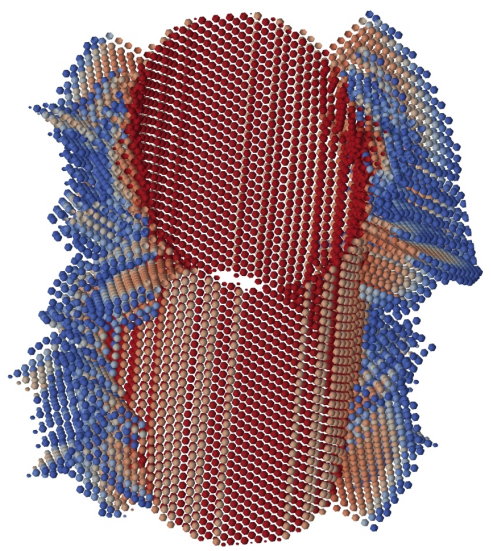

Figure 14: Formation of dislocation loops and surface defects around the cylindrical hole of (a) $1.5 \mathrm{~nm}$ radius and (b) $3 \mathrm{~nm}$ radius, loaded under uniaxial tension (defects have been identified and color-coded by the centrosymmetry parameter Kelchner et al. (1998); arrows indicate the loading direction).

interactions in nano-rods, whiskers, or nano-trusses) or if interesting surface effects appear localized on small fractions of the entire surface of a sample (such as growing voids or cracks reaching the surface), then full atomistic resolution on all surfaces of the sample from the outset is a computational overkill (in particular in small-scale structures with an abundance of free surfaces). This is exactly where coarse-graining techniques such as the ones presented here gain importance, since they allow us to restrict full atomistic resolution to where it is required.

\section{Acknowledgements}

The authors gratefully acknowledge support from the National Science Foundation (NSF) through award CMMI123436.

\section{References}

Abraham, F.F., Broughton, J.Q., Bernstein, N., Kaxiras, E., 1998. Spanning the continuum to quantum length scales in a dynamic simulation of brittle fracture. EPL (Europhysics Letters) 44, 783. URL: http: //stacks . iop. org/0295-5075/44/i=6/a=783.

Agrawal, R., Peng, B., Gdoutos, E.E., Espinosa, H.D., 2008. Elasticity size effects in zno nanowiresa combined experimentalcomputational approach. Nano Letters 8, 3668-3674. URL: http://dx.doi.org/10.1021/nl801724b, doi:10.1021/nl801724b, arXiv:http://dx.doi.org/10.1021/nl801724b.

Amelang, J.S., Venturini, G.N., Kochmann, D.M., 2015. Summation rules for a fully-nonlocal energy-based quasicontinuum method. J. Mech. Phys. Solids. URL: http://www.sciencedirect.com/science/article/pii/S0022509615000630\#, doi:10.1016/j.jmps. 2015.03.007. accepted for publication.

Ariza, M., Romero, I., Ponga, M., Ortiz, M., 2012. Hotqc simulation of nanovoid growth under tension in copper. International Journal of Fracture 174, 75-85. URL: http://dx.doi.org/10.1007/s10704-011-9660-4, doi:10.1007/s10704-011-9660-4.

Asthana, A., Momeni, K., Prasad, A., Yap, Y.K., Yassar, R.S., 2011. In situ observation of size-scale effects on the mechanical properties of zno nanowires. Nanotechnology 22, 265712. URL: http://stacks.iop.org/0957-4484/22/i=26/a=265712.

Beex, L., Peerlings, R., Geers, M., 2014. Central summation in the quasicontinuum method. Journal of the Mechanics and Physics of Solids 70, 242 - 261. URL: http://www.sciencedirect.com/science/article/pii/S0022509614001100, doi:http://dx.doi.org/10. $1016 / j \cdot j m p s .2014 .05 .019$.

Belytschko, T., Xiao, S.P., 2003. Coupling methods for continuum model with molecular model. International Journal for Multiscale Computational Engineering 1, 115-126.

Biener, J., Hodge, A.M., Hamza, A.V., 2007. Deformation behavior of nanoporous metals. Lawrence Livermore Laboratory Report UCRL-BOOK218519.

Bitzek, E., Koskinen, P., Gähler, F., Moseler, M., Gumbsch, P., 2006. Structural relaxation made simple. Phys. Rev. Lett. 97, 170201.

Brenner, S.S., 1956. Tensile strength of whiskers. Journal of Applied Physics 27, 1484-1491.

Brenner, S.S., 1957. Plastic deformation of copper and silver whiskers. Journal of Applied Physics 28, $1023-1026$.

Brinckmann, S., Mahajan, D.K., Hartmaier, A., 2012. A scheme to combine molecular dynamics and dislocation dynamics. Modelling and Simulation in Materials Science and Engineering 20, 045001. URL: http://stacks . iop. org/0965-0393/20/i=4/a=045001.

Broughton, J.Q., Abraham, F.F., Bernstein, N., Kaxiras, E., 1999. Concurrent coupling of length scales: Methodology and application. Phys. Rev. B 60, 2391-2403. URL: http://link.aps.org/doi/10.1103/PhysRevB.60.2391, doi:10.1103/PhysRevB.60.2391. 
Burek, M.J., Greer, J.R., 2010. Fabrication and microstructure control of nanoscale mechanical testing specimens via electron beam lithography and electroplating. Nano letters 10,69-76.

Chen, C., Shi, Y., Zhang, Y., Zhu, J., Yan, Y., 2006. Size dependence of young's modulus in zno nanowires. Phys. Rev. Lett. 96, 075505. URL: http://link.aps.org/doi/10.1103/PhysRevLett.96.075505, doi:10.1103/PhysRevLett.96.075505.

Chen, N., Xie, Y., Liu, F., Ye, X., Shi, W., 2013. A study of the size-dependent elastic properties of cdse nanowires. Computational Materials Science 77, 245 - 249. URL: http://www.sciencedirect.com/science/article/pii/S0927025613002243, doi:http://dx.doi. org/10.1016/j.commatsci.2013.04.052.

Chung, P.W., 2004. Computational method for atomistic homogenization of nanopatterned point defect structures. International Journal for Numerical Methods in Engineering 60, 833-859. URL: http://dx.doi .org/10.1002/nme.989, doi:10.1002/nme.989.

Clayton, J.D., Chung, P.W., 2006. An atomistic-to-continuum framework for nonlinear crystal mechanics based on asymptotic homogenization. Journal of the Mechanics and Physics of Solids 54, 1604-1639. URL: http://www.sciencedirect.com/science/article/pii/ S0022509606000408, doi:10.1016/j.jmps.2006.02.004.

Curtin, W.A., Miller, R.E., 2003. Atomistic/continuum coupling in computational materials science. Modelling and Simulation in Materials Science and Engineering 11, R33. URL: http://stacks.iop.org/0965-0393/11/i=3/a=201.

Dai, X.D., Kong, Y., Li, J.H., Liu, B.X., 2006. Extended finnis-sinclair potential for bcc and fcc metals and alloys. Journal of Physics: Condensed Matter 18, 4527-4542.

Daw, M.S., Baskes, M.I., 1984. Embedded-atom method: Derivation and application to impurities, surfaces, and other defects in metals. Phys. Rev. B 29, 6443-6453.

Dehm, G., 2009. Miniaturized single-crystalline fcc metals deformed in tension: New insights in size-dependent plasticity. Progress in Materials Science 54, 664-688.

Derlet, P., Hasnaoui, A., Van Swygenhoven, H., 2003. Atomistic simulations as guidance to experiments. Scripta Materialia 49, $629-635$.

Dobson, M., Luskin, M., 2009. An analysis of the effect of ghost force oscillation on quasicontinuum error. ESAIM: Mathematical Modelling and Numerical Analysis 43, 591-604.

Dupuy, L.M., Tadmor, E.B., Miller, R.E., Phillips, R., 2005. Finite-temperature quasicontinuum: Molecular dynamics without all the atoms. Physical Review Letters 95, 060202.

Eidel, B., Stukowski, A., 2009. A variational formulation of the quasicontinuum method based on energy sampling in clusters. J. Mech. Phys. Solids 57, 87-108.

Espanol, M.I., Kochmann, D.M., Conti, S., Ortiz, M., 2013. A gamma-convergence analysis of the quasicontinuum method. SIAM Multiscale Modeling and Simulation 11, 766-794.

Fang, C., Yang, X., 2014. Study of nanocontact and incipient nanoscratch process using the quasicontinuum method. Materials Science and Engineering: A 600, 221 - 230. URL: http://www.sciencedirect.com/science/article/pii/S0921509314001671, doi:http:// dx.doi.org/10.1016/j.msea.2014.02.027.

Gill, S.P.A., 2007. The effect of surface-stress on the concentration of stress at nanoscale surface flaws. International Journal of Solids and Structures 44, 7500 - 7509. URL: http://www.sciencedirect.com/science/article/pii/S0020768307002004, doi:http://dx. doi.org/10.1016/j.ijsolstr.2007.04.018.

Greer, J.R., De Hosson, Jeff Th.M., J.T., 2011. Plasticity in small-sized metallic systems: Intrinsic versus extrinsic size effect. Progress in Materials Science 56, 654-724. doi:10.1016/j.pmatsci.2011.01.005.

Greer, J.R., Kim, J.Y., Burek, M.J., 2009. The in-situ mechanical testing of nanoscale single-crystalline nanopillars. Journal of Materials 61, 19-25. Greer, J.R., Nix, W.D., 2006. Nanoscale gold pillars strengthened through dislocation starvation. Physical Review B 73, 1-6.

Greer, J.R., Oliver, W.C., Nix, W.D., 2005. Size dependence of mechanical properties of gold at the micron scale in the absence of strain gradients. Acta Materialia 53, 1821-1830.

Grekov, M., Yazovskaya, A., 2013. Surface stress in an elastic plane with a nearly circular hole, in: Altenbach, H., Morozov, N.F. (Eds.), Surface Effects in Solid Mechanics. Springer Berlin Heidelberg. volume 30 of Advanced Structured Materials, pp. 81-94. URL: http://dx.doi. org/10.1007/978-3-642-35783-1_7, doi:10.1007/978-3-642-35783-1_7.

Gunzburger, M., Zhang, Y., 2010. A quadrature-rule type approximation to the quasi-continuum method. Multiscale Model. Simul. 8, 571-590.

Inglesfield, J., 1995. Theory of surface structure and bonding, North-Holland. volume 4 of Cohesion and Structure, pp. 63 119. URL: http://www.sciencedirect.com/science/article/pii/S0922772506800030, doi:http://dx.doi.org/10.1016/ S0922-7725(06) 80003-0.

Iyer, M., Gavini, V., 2011. A field theoretical approach to the quasi-continuum method. Journal of the Mechanics and Physics of Solids 59, $1506-$ 1535. URL: http://www.sciencedirect.com/science/article/pii/S0022509610002425, doi:10.1016/j.jmps.2010.12.002.

Jennings, A., Burek, M., Greer, J., 2010. Microstructure versus size: Mechanical properties of electroplated single crystalline cu nanopillars. Physical Review Letters , 135503.

Kelchner, C.L., Plimpton, S.J., Hamilton, J.C., 1998. Dislocation nucleation and defect structure during surface indentation. Phys. Rev. B 58, 11085-11088.

Kiener, D., Grosinger, W., Dehm, G., Pippan, R., 2008. A further step towards an understanding of size-dependent crystal plasticity: In situ tension experiments of miniaturized single-crystal copper samples. Acta Materialia 56, 580-592.

Kiener, D., Motz, C., Dehm, G., Pippan, R., 2009. Overview on established and novel FIB based miniaturized mechanical testing using in-situ SEM. International Journal of Materials Research (formerly Zeitschrift fuer Metallkunde) 100, 1074-1087.

Kiener, D., Motz, C., Schoberl, T., Jenko, M., Dehm, G., 2006. Determination of Mechanical Properties of Copper at the Micron Scale. Advanced Engineering Materials 8, 1119-1125.

Kim, J.Y.K.Y., Jang, D., Greer, J.R., 2011. Strength dependence on orientation, size, and deformation path in molybdenum nano-pillars. International Journal of Plasticity .

Kim, W.K., Luskin, M., Perez, D., Voter, A.F., Tadmor, E.B., 2014. Hyper-qc: An accelerated finite-temperature quasicontinuum method using hyperdynamics. J. Mech. Phys. Solids 63, 94-112. doi:http://dx.doi.org/10.1016/j.jmps.2013.10.001.

Kittel, C., 1996. Introduction to Solid State Physics. Wiley, New York 
Knap, J., Ortiz, M., 2001. An analysis of the quasicontinuum method. J. Mech. Phys. Solids 49, 1899-1923.

Kochmann, D., Venturini, G., 2014. A meshless quasicontinuum method based on local maximum-entropy interpolation. Model. Simul. Mater. Sci. Eng. 22, 034007.

Kulkarni, Y., Knap, J., Ortiz, M., 2008. A variational approach to coarse graining of equilibrium and non-equilibrium atomistic description at finite temperature. Journal of the Mechanics and Physics of Solids 56, 1417-1449.

Liu, W.K., Park, H.S., Q., D., Karpov, E.G., Kadowaki, H., Wagner, G.J., 2006. Bridging scale methods for nanomechanics and materials. Computer Methods in Applied Mechanics and Engineering 195, 1407-1421. URL: http://www.sciencedirect.com/science/article/ pii/S0045782505002884, doi:10.1016/j.cma.2005.05.042.

Maass, R., Van Petegem, S., Grolimund, D., Van Swygenhoven, H., Kiener, D., Dehm, G., 2008. Crystal rotation in cu single crystal micropillars: In situ laue and electron backscatter diffraction. Applied Physics Letters 92.

Marian, J., Venturini, G., Hansen, B.L., Knap, J., Ortiz, M., Campbell, G.H., 2010. Finite-temperature extension of the quasicontinuum method using langevin dynamics: entropy losses and analysis of errors. Modelling and Simulation in Materials Science and Engineering $18,015003$.

Miller, R., Ortiz, M., Phillips, R., Shenoy, V., Tadmor, E., 1998. Quasicontinuum models of fracture and plasticity. Engineering Fracture Mechanics $61,427-444$.

Miller, R.E., Shenoy, V.B., 2000. Size-dependent elastic properties of nanosized structural elements. Nanotechnology 11, 139. URL: http: //stacks. iop.org/0957-4484/11/i=3/a=301.

Miri, A.K., Avazmohammadi, R., Yang, F., 2011. Effect of surface stress on the deformation of an elastic half-plane containing a nano-cylindrical hole under a surface loading. Journal of Computational and Theoretical Nanoscience 8, 231-236. URL: http://www. ingentaconnect. com/content/asp/jctn/2011/00000008/00000002/art00012, doi:doi:10.1166/jctn.2011.1683.

Mishin, Y., Mehl, M.J., Papaconstantopoulos, D.A., Voter, A.F., Kress, J.D., 2001. Structural stability and lattice defects in copper: Ab initio, tightbinding, and embedded-atom calculations. Phys. Rev. B 63, 224106. URL: http://link.aps.org/doi/10.1103/PhysRevB.63.224106, doi:10.1103/PhysRevB.63.224106.

Montemayor, L.C., Meza, L.R., Greer, J.R., 2014. Design and fabrication of hollow rigid nanolattices via two-photon lithography. Advanced Engineering Materials 16, 184-189. URL: http://dx.doi.org/10.1002/adem.201300254, doi:10.1002/adem.201300254.

Nair, A., Warner, D., Hennig, R., Curtin, W., 2010. Coupling quantum and continuum scales to predict crack tip dislocation nucleation. Scripta Materialia 63, 1212 - 1215. URL: http://www.sciencedirect.com/science/article/pii/S1359646210005750, doi:10.1016/j. scriptamat. 2010.08.038.

Norfleet, D., Dimiduk, D., Polasik, S., Uchic, M., Mills, M., 2008. Dislocation structures and their relationship to strength in deformed nickel microcrystals. Acta Materialia 56, 2988-3001.

Park, H.S., Karpov, E.G., Liu, W.K., Klein, P.A., 2005. The bridging scale for two-dimensional atomistic/continuum coupling. Philosophical Magazine 85, 79-113. URL: http://www.ingentaconnect.com/content/tandf/tphm/2005/00000085/00000001/art00005, doi:doi: 10.1080/14786430412331300163.

Parthasarathy, T., Rao, S.I., Dimiduk, D., Uchic, M., Trinkle, D., 2007. Contribution to size effect of yield strength from the stochastics of dislocation source lengths in finite samples. Scripta Materialia 56, 313-316.

Poncharal, P., Wang, Z.L., Ugarte, D., de Heer, W.A., 1999. Electrostatic deflections and electromechanical resonances of carbon nanotubes. Science 283, 1513-1516. URL: http://www.sciencemag.org/content/283/5407/1513.abstract, doi:10.1126/science.283.5407. 1513, arXiv:http://www.sciencemag.org/content/283/5407/1513.full.pdf.

Pugno, N., Aifantis, E., 2011. A note on the transition from nano- to mega-mechanics: The role of the stress quantization. Journal of the Mechanical Behavior of Materials 19,1-104. doi:10.1515/JMBM.2009.19.1.31.

Rao, S.I., Dimiduk, D., Parthasarathy, T., Uchic, M., Tang, M., Woodward, C., 2008. Athermal mechanisms of size-dependent crystal flow gleaned from three-dimensional discrete dislocation simulations. Acta Materialia 56, 3245-3259.

Rao, S.I., Dimiduk, D., Tang, M., Parthasarathy, T.A., Uchic, M.D., Woodward, C., 2007. Estimating the strength of single-ended dislocation sources in micron-sized single crystals. Philosophical Magazine 87, 4777-4794.

Rous, P., 1995. Surface crystallography: The experimental data base, North-Holland. volume 4 of Cohesion and Structure, pp. 1 - 61. URL: http://www.sciencedirect.com/science/article/pii/S0922772506800029, doi:http://dx.doi.org/10.1016/ S0922-7725(06)80002-9.

Rudd, R.E., Broughton, J.Q., 2005a. Coarse-grained molecular dynamics: Nonlinear finite elements and finite temperature. Physical Review B 72, 144104.

Rudd, R.E., Broughton, J.Q., 2005b. Coarse-grained molecular dynamics: Nonlinear finite elements and finite temperature. Phys. Rev. B 72, 144104. URL: http://link.aps.org/doi/10.1103/PhysRevB.72.144104, doi:10.1103/PhysRevB.72.144104.

Schaedler, T.A., Jacobsen, A.J., Torrents, A., Sorensen, A.E., Lian, J., Greer, J.R., Valdevit, L., Carter, W.B., 2011. Ultralight metallic microlattices. Science 334, 962-965.

Shan, Z.W., Li, J., Cheng, Y., Minor, A.M., Syed Asif, S.A., Warren, O.L., Ma, E., 2008. Plastic flow and failure resistance of metallic glass: Insight from in situ compression of nanopillars. Physical Review B 77, 1-6.

Shenoy, V., Miller, R., Tadmor, E., Rodney, D., Phillips, R., Ortiz, M., 1999a. An adaptive finite element approach to atomic-scale mechanicsthe quasicontinuum method. Journal of the Mechanics and Physics of Solids 47, 611-642. URL: http://www.sciencedirect.com/science/ article/pii/S0022509698000519, doi:http://dx.doi.org/10.1016/S0022-5096(98)00051-9.

Shenoy, V., Shenoy, V., Phillips, R., 1999b. Finite temperature quasicontinuum methods. Materials Research Society Symposium Proceedings 538, 465471.

Shenoy, V.B., Miller, R., Tadmor, E.B., Phillips, R., Ortiz, M., 1998. Quasicontinuum models of interfacial structure and deformation. Phys. Rev. Lett. 80, 742-745

Shephard, M.S., Picu, C., Datta, D.K., 2004. Composite grid atomistic continuum method: An adaptive approach to bridge continuum with atomistic analysis. International Journal for Multiscale Computational Engineering 2.

Shilkrot, L., Miller, R.E., Curtin, W.A., 2004. Multiscale plasticity modeling: coupled atomistics and discrete dislocation mechanics. Journal of the Mechanics and Physics of Solids 52, $755-787$ 
Simmons, G., Wang, H., 1971. Single Crystal Elastic Constants and Calculated Aggregate Properties. MIT Press, Camebridge, MA.

Sorensen, M.R., Voter, A.F., 2000. Temperature-accelerated dynamics for simulation of infrequent events. The Journal of Chemical Physics 112.

Suryanarayana, P., 2011. Coarse-graining Kohn-Sham density functional theory. Ph.D. thesis. California Institute of Technology.

Tadmor, E., Legoll, F., Kim, W., Dupuy, L., Miller, R., 2013. Finite-temperature quasi-continuum. Applied Mechanics Reviews 65, 010803. URL: http://hal .archives-ouvertes.fr/hal-00849048, doi:10.1115/1.4023013.

Tadmor, E., Ortiz, M., Phillips, R., 1996. Quasicontinuum analysis of defects in solids. Philos. Mag. A 73, $1529-1563$.

Taylor, G.F., 1924. A method of drawing metallic filaments and a discussion of their properties and uses. Phys. Rev. 23, 655-660.

Todd, B., Lynden-Bell, R., 1993. Surface and bulk properties of metals modelled with sutton-chen potentials. Surface Science 281, 191 - 206. URL: http://www.sciencedirect.com/science/article/pii/003960289390868K, doi:http://dx.doi.org/10.1016/ 0039-6028 (93) 90868-K.

Venturini, G., Wang, K., Romero, I., Ariza, M., Ortiz, M., 2014. Atomistic long-term simulation of heat and mass transport. Journal of the Mechanics and Physics of Solids 73, 242 - 268. URL: http://www.sciencedirect.com/science/article/pii/S002250961400194X, doi:http://dx.doi.org/10.1016/j.jmps.2014.09.008.

Voter, A., 1997. A method for accelerating the molecular dynamics simulation of infrequent events. The Journal of Chemical Physics 106, 4665-4677. URL: http://link.aip.org/link/?JCP/106/4665/1, doi:10.1063/1.473503.

Voter, A.F., 1998. Parallel replica method for dynamics of infrequent events. Phys. Rev. B 57, R13985-R13988. URL: http://link. aps . org/ doi/10.1103/PhysRevB.57.R13985, doi:10.1103/PhysRevB.57.R13985.

Voter, A.F., Montalenti, F., Germann, T.C., 2002. Extending the time scale in atomistic simulation of materials. Annual Review of Materials Research 32, 321-346. URL: http://www.annualreviews.org/ doi/abs/10.1146/annurev.matsci.32.112601.141541, doi:10.1146/annurev.matsci.32.112601.141541, arXiv:http://www.annualreviews.org/doi/pdf/10.1146/annurev.matsci.32.112601.141541.

Wong, E.W., Sheehan, P.E., Lieber, C.M., 1997. Nanobeam mechanics: Elasticity, strength, and toughness of nanorods and nanotubes. Science 277, 1971-1975. URL: http://www.sciencemag.org/content/277/5334/1971.abstract, doi:10.1126/science.277.5334.1971, arXiv:http://www.sciencemag.org/content/277/5334/1971.full.pdf.

Yang, Q., E., B., To, A., 2013. Multiresolution molecular mechanics: Statics. Computer Methods in Applied Mechanics and Engineering 258, 26 - 38. URL: http://www.sciencedirect.com/science/article/pii/S004578251300025X, doi:http://dx.doi.org/10.1016/j. cma. 2013.01.014.

Zhu, T., Li, J., Ogata, S., Yip, S., 2009. Mechanics of ultra-strength materials. MRS Bulletin 34, 167-172.

Zhu, Y., Qin, Q., Xu, F., Fan, F., Ding, Y., Zhang, T., Wiley, B.J., Wang, Z.L., 2012. Size effects on elasticity, yielding, and fracture of silver nanowires: In situ experiments. Phys. Rev. B 85, 045443. URL: http://link.aps.org/doi/10.1103/PhysRevB.85.045443, doi:10. 1103/PhysRevB. 85.045443. 


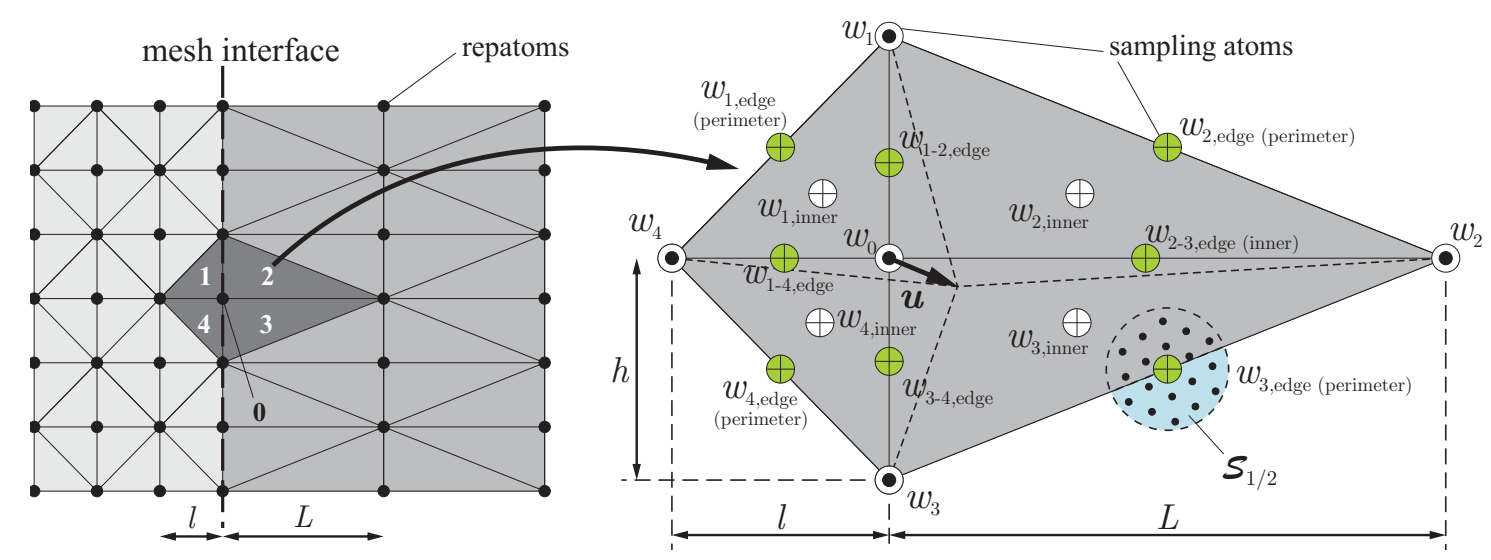

Figure A.15: Two-dimensional scenario to determine the residual force on a sharp mesh interface with a zoom into the four elements adjacent to repatom 0 at the center. A residual force $\widetilde{\boldsymbol{F}}_{0}$ on repatom 0 emerges if the body can seemingly lower its energy by a displacement $\boldsymbol{u}$. Repatoms have a solid filling, sampling atoms have open circles; crosses denote sampling atoms which undergo Cauchy-Born deformation (green sampling atoms on element edges only exist in the new second-order summation rule). The sampling atom on the outer edge of element 3 illustrates a typical atomic neighborhood (the blue semicircle of interaction is denoted by $\mathcal{S}_{1 / 2}$ in the derivations).

\section{Appendix A. Residual force at a sharp mesh interface in two dimensions}

Residual force artifacts arise in non-uniform QC meshes. To illustrate this phenomenon, let us consider a sharp interface in a semi-structured two-dimensional QC mesh with an affine interpolation (as used throughout this paper). Fig. A.15 illustrates such a scenario with elements coarsened in the horizontal direction by a factor of $L / l$. We will determine the residual force acting on a repatom located at the interface (here labeled 0 ) by analyzing the patch of its four adjacent elements (shown in dark gray). A residual force on repatom 0 arises if the summation rule furnishes the system with the possibility to reduce its (approximated) total potential energy by displacing the repatom 0 . To specialize to the new first- and second-order summation rules of (Amelang et al., 2015), we assume that all repatoms are sampling atoms, and that every element contains one sampling atom in its interior. With appropriate weights, this represents the new first-order rule $\left(0,1^{*}\right)$. In order to study the new second-order rule $\left(0,1^{*}, 3^{*}\right)$ we include additional sampling atoms, viz. one per element edge (as shown in Fig. A.15).

Let us derive the residual force acting on the interface sampling atom 0 . The (approximated) total potential energy affected by the position $\boldsymbol{x}_{0}$ of the central repatom is given by the summation over all sampling atoms contained in the four adjacent elements $(e=1, \ldots, 4)$, since their atomic neighborhoods depend on $\boldsymbol{x}_{0}$ through interpolation:

$$
\widetilde{V}=\sum_{e=1}^{4} w_{e, \text { inner }} E_{e, \text { inner }}+w_{0} E_{0}+\sum_{i=1}^{4} w_{i} E_{i, \text { vertex }}+\sum_{i=1}^{4} w_{i, \text { edge }} E_{i, \text { edge }}+\sum_{e, f} w_{e-f, \text { edge }} E_{e-f, \text { edge }}
$$

where $E_{e \text {,inner }}$ denotes the energy of the sampling atom located inside element $e, E_{i, \text { vertex }}$ is the energy of outer repatom $i, E_{0}$ is the energy of the central repatom, and $E_{i, \text { edge }}$ and $E_{e-f \text {,edge }}$ are the energies of sampling atoms located on the outer edge of element $i$ and between elements $i$ and $j$, respectively (these sampling atoms exist in the second-order summation rule). $w_{e, \text { inner }}, w_{i}, w_{0}, w_{e, \text { edge }}$, and $w_{e-f \text {,edge }}$ denote the corresponding weights of the sampling atoms, see Fig. A.15. We assume that that inner-element sampling atoms experiences affine neighborhood deformations of Cauchy-Born type, and that the sampling atoms on edges see affine Cauchy-Born deformation on either side of the element edge. The residual force on repatom 0 thus follows as

$$
\widetilde{\boldsymbol{F}}_{0}=-\frac{\partial \widetilde{V}}{\partial \boldsymbol{x}_{0}}=-\sum_{e=1}^{4} \frac{\partial \widetilde{V}}{\partial \boldsymbol{F}_{e}} \cdot \frac{\partial \boldsymbol{F}_{e}}{\partial \boldsymbol{x}_{0}} .
$$

For the chosen geometric example, the deformation gradients $\boldsymbol{F}_{e}$ in elements 1 through 4 all depend on $\boldsymbol{x}_{0}$. Assume the central repatom is displaced to the new location $\boldsymbol{x}_{0}=\left(x_{0}, y_{0}\right)=\boldsymbol{X}+(u, v)$, whereas the remaining repatoms remain 
in the undeformed ground state. Since we use an affine interpolation within each element, the deformation gradients in the four elements are constant and given by

$$
\boldsymbol{F}_{1}=\left(\begin{array}{cc}
1+\frac{u}{l} & -\frac{u}{h} \\
\frac{v}{l} & 1-\frac{v}{h}
\end{array}\right), \quad \boldsymbol{F}_{2}=\left(\begin{array}{cc}
1+\frac{u}{l} & \frac{u}{h} \\
\frac{v}{l} & 1+\frac{v}{h}
\end{array}\right), \quad \boldsymbol{F}_{3}=\left(\begin{array}{cc}
1-\frac{u}{L} & \frac{u}{h} \\
-\frac{v}{L} & 1+\frac{v}{h}
\end{array}\right), \quad \boldsymbol{F}_{4}=\left(\begin{array}{cc}
1-\frac{u}{L} & -\frac{u}{h} \\
-\frac{v}{L} & 1-\frac{v}{h}
\end{array}\right),
$$

where $l$ and $L$ are the horizontal lengths of elements to the left and to the right of the interface, respectively, and $h$ is the common height of all elements (see Fig. A.15). By using element areas $A_{1}=A_{2}=h l / 2$ and $A_{3}=A_{4}=h L / 2$, (A.3) also implies that

$$
\sum_{e=1}^{4} \boldsymbol{F}_{e} A_{e}=\boldsymbol{I} \sum_{e=1}^{4} A_{e} \quad \Rightarrow \quad \sum_{e=1}^{4} \frac{\partial \boldsymbol{F}_{e}}{\partial u} A_{e}=0, \wedge \sum_{e=1}^{4} \frac{\partial \boldsymbol{F}_{e}}{\partial v} A_{e}=0 \quad \Leftrightarrow \quad \sum_{e=1}^{4} \frac{\partial \boldsymbol{F}_{e}}{\partial \boldsymbol{x}_{0}} A_{e}=\mathbf{0}
$$

We note that these relations also hold if we do not start from the undeformed ground state but if instead we start from an affinely-deformed initial state with uniform deformation gradient $\boldsymbol{F}_{0}=$ const. In this case deformation gradients in (A.3) change into $\hat{\boldsymbol{F}}_{i}=\boldsymbol{F}_{0} \boldsymbol{F}_{i}$ with $\boldsymbol{F}_{i}$ from (A.3), so that one arrives at the same final relation in (A.4).

Let us assume the energy $E_{i}$ of any atom $i$ is defined by an interatomic potential of the Embedded Atom Method (Daw and Baskes, 1984), so that (3) applies. The inner-element sampling atoms experience an affine deformation. For conciseness, let us denote by $\mathcal{S}$ the set of all lattice sites contained within a neighborhood of interaction, and $\boldsymbol{R}_{j}$ be the distance vector from any atom to its neighboring lattice site $j(j \in \mathcal{S})$. For an EAM potential this leads to

$$
E_{e, \text { inner }}=\sum_{j \in \mathcal{S}} \frac{1}{2} \Phi\left(\left|\boldsymbol{F}_{e} \boldsymbol{R}_{j}\right|\right)+\mathcal{F}\left(\rho_{e}\right), \quad \rho_{e}=\sum_{j \in \mathcal{S}} \phi\left(\left|\boldsymbol{F}_{e} \boldsymbol{R}_{j}\right|\right) .
$$

Consequently, we obtain the contribution to the residual force from the sampling atom within element $e$ via

$$
\frac{\partial E_{e, \text { inner }}}{\partial \boldsymbol{x}_{0}}=\frac{\partial E_{e, \text { inner }}}{\partial \boldsymbol{F}_{e}} \cdot \frac{\partial \boldsymbol{F}_{e}}{\partial \boldsymbol{x}_{0}}=\sum_{j \in \mathcal{S}}\left[\frac{1}{2} \Phi^{\prime}\left(\left|\boldsymbol{F}_{e} \boldsymbol{R}_{j}\right|\right)+\mathcal{F}^{\prime}\left(\rho_{e}\right) \phi^{\prime}\left(\left|\boldsymbol{F}_{e} \boldsymbol{R}_{j}\right|\right)\right] \frac{\boldsymbol{F}_{e} \boldsymbol{R}_{j} \otimes \boldsymbol{R}_{j}}{\left|\boldsymbol{F}_{e} \boldsymbol{R}_{j}\right|} \cdot \frac{\partial \boldsymbol{F}_{e}}{\partial \boldsymbol{x}_{0}} .
$$

Similarly, we can consider sampling atoms on element edges which are assumed to experience an affine deformation on either side of the interface. Thus, the energy of a sampling atom on the edge between elements $a$ and $b$ involves half an atomic neighborhood $\mathcal{S}_{1 / 2}$ within either of the two adjacent elements. In addition, for centrosymmetric lattices, summation over any (arbitrarily oriented) semicircle of interaction $\mathcal{S}_{1 / 2}$ yields the same answer, viz.

$$
E_{a-b, \text { edge }}=\sum_{j \in \mathcal{S}_{1 / 2}} \frac{1}{2} \Phi\left(\left|\boldsymbol{F}_{a} \boldsymbol{R}_{j}\right|\right)+\sum_{j \in \mathcal{S}_{1 / 2}} \frac{1}{2} \Phi\left(\left|\boldsymbol{F}_{b} \boldsymbol{R}_{j}\right|\right)+\mathcal{F}\left(\rho_{e}\right), \quad \rho_{e}=\sum_{j \in \mathcal{S}_{1 / 2}} \phi\left(\left|\boldsymbol{F}_{a} \boldsymbol{R}_{j}\right|\right)+\sum_{j \in \mathcal{S}_{1 / 2}} \phi\left(\left|\boldsymbol{F}_{b} \boldsymbol{R}_{j}\right|\right) .
$$

Here, the contribution to the residual force depends on the location of the sampling atom. In general, we have

$$
\begin{aligned}
\frac{\partial E_{a-b, \text { edge }}}{\partial \boldsymbol{x}_{0}}= & \frac{\partial E_{a-b, \text { edge }}}{\partial \boldsymbol{F}_{a}} \cdot \frac{\partial \boldsymbol{F}_{a}}{\partial \boldsymbol{x}_{0}}+\frac{\partial E_{a-b, \text { edge }}}{\partial \boldsymbol{F}_{b}} \cdot \frac{\partial \boldsymbol{F}_{b}}{\partial \boldsymbol{x}_{0}} \\
= & \sum_{j \in \mathcal{S}_{1 / 2}}\left[\frac{1}{2} \Phi^{\prime}\left(\left|\boldsymbol{F}_{a} \boldsymbol{R}_{j}\right|\right)+\mathcal{F}^{\prime}\left(\rho_{a}\right) \phi^{\prime}\left(\left|\boldsymbol{F}_{a} \boldsymbol{R}_{j}\right|\right)\right] \frac{\boldsymbol{F}_{a} \boldsymbol{R}_{j} \otimes \boldsymbol{R}_{j}}{\left|\boldsymbol{F}_{a} \boldsymbol{R}_{j}\right|} \cdot \frac{\partial \boldsymbol{F}_{a}}{\partial \boldsymbol{x}_{0}} \\
& +\sum_{j \in \mathcal{S}_{1 / 2}}\left[\frac{1}{2} \Phi^{\prime}\left(\left|\boldsymbol{F}_{b} \boldsymbol{R}_{j}\right|\right)+\mathcal{F}^{\prime}\left(\rho_{b}\right) \phi^{\prime}\left(\left|\boldsymbol{F}_{b} \boldsymbol{R}_{j}\right|\right)\right] \frac{\boldsymbol{F}_{b} \boldsymbol{R}_{j} \otimes \boldsymbol{R}_{j}}{\left|\boldsymbol{F}_{b} \boldsymbol{R}_{j}\right|} \cdot \frac{\partial \boldsymbol{F}_{b}}{\partial \boldsymbol{x}_{0}}
\end{aligned}
$$

If both elements $a$ and $b$ lie within the patch of four elements, then the above form holds as written. If element $a$ or $b$ lies outside the patch of elements, then the respective term in (A.8) vanishes since $\partial \boldsymbol{F}_{e} / \partial \boldsymbol{x}_{0}=\mathbf{0}$ if element $e$ is not adjacent to node 0 . When considering residual forces in an affinely-strained ground state with uniform deformation gradient $\boldsymbol{F}_{0}$, then relations (A.6) and (A.8) hold if we set $\boldsymbol{F}_{e}=\boldsymbol{F}_{0}$ for all $e=1, \ldots, 4$. For example, the case of an affine ground state reduces (A.8) to

$$
\left.\frac{\partial E_{a-b, \text { edge }}}{\partial \boldsymbol{x}_{0}}\right|_{\boldsymbol{F}_{e}=\boldsymbol{F}_{0}}=\sum_{j \in \mathcal{S}_{1 / 2}}\left[\frac{1}{2} \Phi^{\prime}\left(\left|\boldsymbol{F}_{0} \boldsymbol{R}_{j}\right|\right)+\mathcal{F}^{\prime}\left(\rho_{0}\right) \phi^{\prime}\left(\left|\boldsymbol{F}_{0} \boldsymbol{R}_{j}\right|\right)\right] \frac{\boldsymbol{F}_{0} \boldsymbol{R}_{j} \otimes \boldsymbol{R}_{j}}{\left|\boldsymbol{F}_{0} \boldsymbol{R}_{j}\right|} \cdot\left(\frac{\partial \boldsymbol{F}_{a}}{\partial \boldsymbol{x}_{0}}+\frac{\partial \boldsymbol{F}_{b}}{\partial \boldsymbol{x}_{0}}\right), \quad \rho_{0}=\sum_{j \in \mathcal{S}} \phi\left(\left|\boldsymbol{F}_{0} \boldsymbol{R}_{j}\right|\right) .
$$


In contrast, if the sampling atom is located on an edge on the perimeter of the patch of elements, then only one of the two semicircles of interaction is affected by $\boldsymbol{x}_{0}$ (assume it is element $a$ ), which yields for the affine ground state

$$
\left.\frac{\partial E_{a-b, \text { edge }}}{\partial \boldsymbol{x}_{0}}\right|_{\boldsymbol{F}_{e}=\boldsymbol{F}_{0}}=\sum_{j \in \mathcal{S}_{1 / 2}}\left[\frac{1}{2} \Phi^{\prime}\left(\left|\boldsymbol{F}_{0} \boldsymbol{R}_{j}\right|\right)+\mathcal{F}^{\prime}\left(\rho_{0}\right) \phi^{\prime}\left(\left|\boldsymbol{F}_{0} \boldsymbol{R}_{j}\right|\right)\right] \frac{\boldsymbol{F}_{0} \boldsymbol{R}_{j} \otimes \boldsymbol{R}_{j}}{\left|\boldsymbol{F}_{0} \boldsymbol{R}_{j}\right|} \cdot \frac{\partial \boldsymbol{F}_{a}}{\partial \boldsymbol{x}_{0}}, \quad \rho_{0}=\sum_{j \in \mathcal{S}} \phi\left(\left|\boldsymbol{F}_{0} \boldsymbol{R}_{j}\right|\right) .
$$

Altogether, for the shown patch of four elements the residual force on the central repatom 0 in an affinely deformed configuration with uniform deformation gradient $\boldsymbol{F}_{0}$ is given by summing the contribution from all $E_{i, \text { inner }}$ and $E_{i \text {,edge }}$

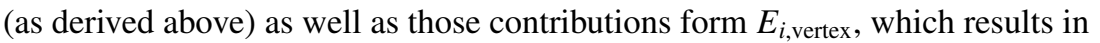

$$
\begin{aligned}
& \widetilde{\boldsymbol{F}}_{0}\left(\boldsymbol{x}_{0}\right)=-\sum_{e=1}^{4} w_{e, \text { inner }} \sum_{j \in \mathcal{S}}\left[\frac{1}{2} \Phi^{\prime}\left(\left|\boldsymbol{F}_{0} \boldsymbol{R}_{j}\right|\right)+\mathcal{F}^{\prime}\left(\rho_{0}\right) \phi^{\prime}\left(\left|\boldsymbol{F}_{0} \boldsymbol{R}_{j}\right|\right)\right] \frac{\boldsymbol{F}_{0} \boldsymbol{R}_{j} \otimes \boldsymbol{F}_{0} \boldsymbol{R}_{j}}{\left|\boldsymbol{F}_{0} \boldsymbol{R}_{j}\right|} \cdot \frac{\partial \boldsymbol{F}_{e}}{\partial \boldsymbol{x}_{0}} \\
& -\sum_{e, f} w_{e-f, \text { edge (inner) }} \sum_{j \in \mathcal{S}_{1 / 2}}\left[\frac{1}{2} \Phi^{\prime}\left(\left|\boldsymbol{F}_{0} \boldsymbol{R}_{j}\right|\right)+\mathcal{F}^{\prime}\left(\rho_{0}\right) \phi^{\prime}\left(\left|\boldsymbol{F}_{0} \boldsymbol{R}_{j}\right|\right)\right] \frac{\boldsymbol{F}_{0} \boldsymbol{R}_{j} \otimes \boldsymbol{F}_{0} \boldsymbol{R}_{j}}{\left|\boldsymbol{F}_{0} \boldsymbol{R}_{j}\right|} \cdot\left(\frac{\partial \boldsymbol{F}_{e, 1}}{\partial \boldsymbol{x}_{0}}+\frac{\partial \boldsymbol{F}_{f, 2}}{\partial \boldsymbol{x}_{0}}\right) \\
& -\sum_{e=1}^{4} w_{e, \text { edge (perimeter) }} \sum_{j \in \mathcal{S}_{1 / 2}}\left[\frac{1}{2} \Phi^{\prime}\left(\left|\boldsymbol{F}_{0} \boldsymbol{R}_{j}\right|\right)+\mathcal{F}^{\prime}\left(\rho_{0}\right) \phi^{\prime}\left(\left|\boldsymbol{F}_{0} \boldsymbol{R}_{j}\right|\right)\right] \frac{\boldsymbol{F}_{0} \boldsymbol{R}_{j} \otimes \boldsymbol{F}_{0} \boldsymbol{R}_{j}}{\left|\boldsymbol{F}_{0} \boldsymbol{R}_{j}\right|} \cdot \frac{\partial \boldsymbol{F}_{e}}{\partial \boldsymbol{x}_{0}} \\
& -\left(w_{4} \sum_{\boldsymbol{R}_{j} \in\left[0^{\circ} ; \alpha\right)}+w_{1} \sum_{\boldsymbol{R}_{j} \in[\alpha ; \pi / 2)}+w_{0} \sum_{\boldsymbol{R}_{j} \in[\pi / 2 ; \pi)}\right)\left[\frac{1}{2} \Phi^{\prime}\left(\left|\boldsymbol{F}_{0} \boldsymbol{R}_{j}\right|\right)+\mathcal{F}^{\prime}\left(\rho_{0}\right) \phi^{\prime}\left(\left|\boldsymbol{F}_{0} \boldsymbol{R}_{j}\right|\right)\right] \frac{\boldsymbol{F}_{0} \boldsymbol{R}_{j} \otimes \boldsymbol{F}_{0} \boldsymbol{R}_{j}}{\left|\boldsymbol{F}_{0} \boldsymbol{R}_{j}\right|} \cdot \frac{\partial \boldsymbol{F}_{1}}{\partial \boldsymbol{x}_{0}} \\
& -\left(w_{0} \sum_{\boldsymbol{R}_{j} \in\left[0^{\circ} ; \pi / 2\right)}+w_{1} \sum_{\boldsymbol{R}_{j} \in[\pi / 2 ; \pi-\beta)}+w_{2} \sum_{\boldsymbol{R}_{j} \in[\pi-\beta ; \pi)}\right)\left[\frac{1}{2} \Phi^{\prime}\left(\left|\boldsymbol{F}_{0} \boldsymbol{R}_{j}\right|\right)+\mathcal{F}^{\prime}\left(\rho_{0}\right) \phi^{\prime}\left(\left|\boldsymbol{F}_{0} \boldsymbol{R}_{j}\right|\right)\right] \frac{\boldsymbol{F}_{0} \boldsymbol{R}_{j} \otimes \boldsymbol{F}_{0} \boldsymbol{R}_{j}}{\left|\boldsymbol{F}_{0} \boldsymbol{R}_{j}\right|} \cdot \frac{\partial \boldsymbol{F}_{2}}{\partial \boldsymbol{x}_{0}} \\
& -\left(w_{2} \sum_{\boldsymbol{R}_{j} \in\left[0^{\circ} ; \beta\right)}+w_{3} \sum_{\boldsymbol{R}_{j} \in[\beta ; \pi / 2)}+w_{0} \sum_{\boldsymbol{R}_{j} \in[\pi / 2 ; \pi)}\right)\left[\frac{1}{2} \Phi^{\prime}\left(\left|\boldsymbol{F}_{0} \boldsymbol{R}_{j}\right|\right)+\mathcal{F}^{\prime}\left(\rho_{0}\right) \phi^{\prime}\left(\left|\boldsymbol{F}_{0} \boldsymbol{R}_{j}\right|\right)\right] \frac{\boldsymbol{F}_{0} \boldsymbol{R}_{j} \otimes \boldsymbol{F}_{0} \boldsymbol{R}_{j}}{\left|\boldsymbol{F}_{0} \boldsymbol{R}_{j}\right|} \cdot \frac{\partial \boldsymbol{F}_{3}}{\partial \boldsymbol{x}_{0}} \\
& -\left(w_{0} \sum_{\boldsymbol{R}_{j} \in\left[0^{\circ} ; \pi / 2\right)}+w_{4} \sum_{\boldsymbol{R}_{j} \in[\pi / 2-\alpha ; \pi / 2)}+w_{3} \sum_{\boldsymbol{R}_{j} \in[\pi / 2 ; \pi / 2+\alpha)}\right)\left[\frac{1}{2} \Phi^{\prime}\left(\left|\boldsymbol{F}_{0} \boldsymbol{R}_{j}\right|\right)+\mathcal{F}^{\prime}\left(\rho_{0}\right) \phi^{\prime}\left(\left|\boldsymbol{F}_{0} \boldsymbol{R}_{j}\right|\right)\right] \frac{\boldsymbol{F}_{0} \boldsymbol{R}_{j} \otimes \boldsymbol{F}_{0} \boldsymbol{R}_{j}}{\left|\boldsymbol{F}_{0} \boldsymbol{R}_{j}\right|} \cdot \frac{\partial \boldsymbol{F}_{4}}{\partial \boldsymbol{x}_{0}}
\end{aligned}
$$

where $\boldsymbol{R}_{j} \in[x ; y)$ denotes the set of all lattice sites contained within the segment of the circle of interaction ranging from angle $x$ to angle $y$ (counter-clockwise from the horizontal axis) and

$$
\alpha=\arctan (h / l), \quad \beta=\arctan (h / L) .
$$

In the new first- and second-order summation rules of Amelang et al. (2015), we choose weights according to

$$
w_{0}=w_{1}=\ldots=w_{n_{e}}=w_{\text {rep }}
$$

i.e. we assign equal weights $w_{i}=w_{\text {rep }}$ to all sampling atoms on repatoms $(i=0, \ldots, 4)$ so that $(\mathrm{A} .11)$ reduces to

$$
\begin{aligned}
\widetilde{\boldsymbol{F}}_{0}\left(\boldsymbol{x}_{0}\right)=-\sum_{e=1}^{4} & {\left[w_{e, \text { inner }}+\frac{1}{2} w_{e, \text { edge (perimeter) }}+\sum_{i=1}^{2} \frac{1}{2} w_{e, i, \text { edge (inner) }}+\frac{1}{2} w_{\text {rep }}\right] } \\
& \times \sum_{j \in \mathcal{S}}\left[\frac{1}{2} \Phi^{\prime}\left(\left|\boldsymbol{F}_{0} \boldsymbol{R}_{j}\right|\right)+\mathcal{F}^{\prime}\left(\rho_{0}\right) \phi^{\prime}\left(\left|\boldsymbol{F}_{0} \boldsymbol{R}_{j}\right|\right)\right] \frac{\boldsymbol{F}_{0} \boldsymbol{R}_{j} \otimes \boldsymbol{R}_{j}}{\left|\boldsymbol{F}_{0} \boldsymbol{R}_{j}\right|} \cdot \frac{\partial \boldsymbol{F}_{e}}{\partial \boldsymbol{x}_{0}},
\end{aligned}
$$

where $w_{e, i \text {,edge (inner) }}$ denotes the weight of the sampling atom located on the (inner) edge of element $e$ shared with adjacent element $e$. 
As discussed in (Amelang et al., 2015), the undeformed ground state $\left(\boldsymbol{F}_{0}=\boldsymbol{I}\right)$ of an infinite single crystal is characterized by a minimum of the total potential energy. This implies that

$$
\sum_{j \in \mathcal{S}}\left[\frac{1}{2} \Phi^{\prime}\left(\left|\boldsymbol{R}_{j}\right|\right)+\mathcal{F}^{\prime}\left(\rho_{0}(\boldsymbol{I})\right) \phi^{\prime}\left(\left|\boldsymbol{R}_{j}\right|\right)\right] \frac{\boldsymbol{R}_{j} \otimes \boldsymbol{R}_{j}}{\left|\boldsymbol{R}_{j}\right|}=\mathbf{0} .
$$

We see that application of $\boldsymbol{F}_{0}=\boldsymbol{I}$ to (A.14) makes the residual force on the interface repatom in the undeformed ground state (using the new summation rules) vanish for all choices of $l$ and $L$. Any other summation rule (including nodal, cluster, or quadrature rules) whose weights at repatom locations are based on element sizes or geometries does not allow for this reduction, so that they will necessarily produce residual force artifacts. For example, consider a nodal summation rule with $w_{e \text {,inner }}=w_{i \text {,edge }}=0$ applied to the chosen geometry in an arbitrary affinely-strained configuration (with deformation gradient $\boldsymbol{F}_{0}$ ). In this case, insertion of the appropriate nodal weights leads to

$$
\widetilde{\boldsymbol{F}}_{0}\left(\boldsymbol{x}_{0}\right)=-2 w_{r} \frac{L-l}{l L} f\left(\boldsymbol{R}_{1}, \ldots, \boldsymbol{R}_{n} ; \boldsymbol{F}_{0}\right)
$$

with a generally non-vanishing function $f(\cdot)$. Apparently, this force only vanishes if $l=L$, i.e. in a uniform QC mesh. Otherwise, a residual/spurious force artifact (in the horizontal direction) appears at the interface.

Finally, the new first- and second-order summation rules are designed to eliminate not only residual forces in the undeformed ground state but also all spurious force artifacts in any affinely-deformed configuration in centrosymmetric lattices. To this end, weights are chosen such that

$$
w_{e, \text { inner }}+\frac{1}{2} w_{e, \text { edge(perimeter) }}+\sum_{i=1}^{2} \frac{1}{2} w_{e, i, \text { edge (inner) }}+\frac{1}{2} w_{\text {rep }}=0
$$

for all elements $e$. To minimize the energy error, this is achieved by defining $w_{\text {rep }}=\pi r^{2}$, where $r$ denotes the effective atomic interaction distance. In the first-order rule $\left(0,1^{*}\right)$ we have $w_{e, \text { edge }}=0$, and in the second-order rule $\left(0,1^{*}, 4^{*}\right)$ we define $w_{e, \text { edge }}=r\left(L_{e}-2 r\right)$ with $L_{e}$ being the length of element edge $e$, see also the graphical interpretation in Fig. 4. In either of the two rules, the inner-element sampling atom's weight is set to

$$
w_{e, \text { inner }}=n_{e, \text { total }}-\left(\frac{1}{2} w_{\text {rep }}+\frac{1}{2} w_{e, \text { edge(perimeter })}+\sum_{i=1}^{2} \frac{1}{2} w_{e, i \text {,edge }(\text { inner })}\right) .
$$

That is, after assigning weights to all repatoms and to all sampling atoms on edges, the inner-element sampling atoms receive the remaining number of lattice sites within the respective elements as their weights (and $n_{e \text {,total }}$ is proportional to the element area $A_{e}$ ). This leads to a spurious force of

$$
\widetilde{\boldsymbol{F}}_{0}\left(\boldsymbol{x}_{0}\right)=-\sum_{j \in \mathcal{S}}\left[\frac{1}{2} \Phi^{\prime}\left(\left|\boldsymbol{F}_{0} \boldsymbol{R}_{j}\right|\right)+\mathcal{F}^{\prime}\left(\rho_{0}\right) \phi^{\prime}\left(\left|\boldsymbol{F}_{0} \boldsymbol{R}_{j}\right|\right)\right] \frac{\boldsymbol{F}_{0} \boldsymbol{R}_{j} \otimes \boldsymbol{R}_{j}}{\left|\boldsymbol{F}_{0} \boldsymbol{R}_{j}\right|} \cdot \sum_{e=1}^{4} A_{e} \frac{\partial \boldsymbol{F}_{e}}{\partial \boldsymbol{x}_{0}}=\mathbf{0}
$$

Hence, in the undeformed ground state with $\boldsymbol{F}_{0}=\boldsymbol{I}$ the first sum vanishes, whereas in any affinely-deformed state with $\boldsymbol{F}_{0} \neq \boldsymbol{I}$ the second sum vanishes.

In summary, the new first- and second-order summation rules do not produce any residual or spurious force artifacts on the interface repatoms in any affinely-deformed, non-uniform QC mesh. The example shown above can be extended to general (non-sharp) interfaces; the lengthy derivation is omitted here, see (Amelang et al., 2015) for a detailed discussion of that case. 